Алгебра и анализ

Том. 18 (2006), № 1
St. Petersburg Math. J.

Vol. 18 (2007), No. 1, Pages 141-181

S 1061-0022(07)00947-8

Article electronically published on January 24, 2007

\title{
BRANCHING POINTS AREA THEOREMS FOR UNIVALENT FUNCTIONS
}

\author{
S. SHIMORIN
}

\begin{abstract}
Area theorems of a new type are obtained by considering branching point compositions with univalent functions. Such theorems can be formulated both in the form of integral estimates and in the form of Grunsky and Goluzin-type inequalities.
\end{abstract}

\section{§1. INTRODUCTION}

Area methods are among the most important and powerful tools in the theory of conformal mappings. The monograph 8 ] was devoted entirely to these topics; a wide exposition of various forms of area principles can be found also in the classical monographs [5] or [10]. Recent progress in estimating integral means of the derivatives of univalent functions (see [12, 7]) was based on the use of area inequalities combined with techniques of Bergman spaces. This was an inspiration for taking a new look at area methods in the theory of conformal mappings.

The classical polynomial area theorem for univalent functions can be formulated as follows: if $\psi$ is a function univalent in the unit disk $\mathbb{D}$ of the complex plane $\mathbb{C}$, and $p$ is a Laurent polynomial

$$
p(z)=\sum_{n=0}^{m} \frac{p_{n}}{z^{n}}
$$

then the composition $p(\psi)$ written as a Laurent series converging for $z$ near $\mathbb{T}=\partial \mathbb{D}$, namely,

$$
p(\psi(z))=\sum_{k=-\infty}^{+\infty} c_{k} z^{k}
$$

satisfies

$$
[p(\psi), p(\psi)] \leq 0,
$$

where $[\cdot, \cdot]$ is the indefinite inner product defined as follows:

$$
[g, g]=\sum_{k \in \mathbb{Z}} k\left|c_{k}\right|^{2} \quad \text { for } g(z)=\sum_{k \in \mathbb{Z}} c_{k} z^{k} .
$$

Equivalently, (1.1) can be rewritten as

$$
\sum_{k \geq 0} k\left|c_{k}\right|^{2} \leq \sum_{l<0}(-l)\left|c_{l}\right|^{2}
$$

(and this corresponds to a canonical decomposition of the indefinite norm (1.2) into a sum of orthogonal positive and negative parts). The conclusion of the polynomial area theorem remains true if $p$ is an arbitrary rational function with poles in $\psi(\mathbb{D})$ or even a

2000 Mathematics Subject Classification. Primary 30C50.

Key words and phrases. Univalent functions, branching point, area theorems.

(C)2007 American Mathematical Society 
function regular in a neighborhood of $\overline{\mathbb{C}} \backslash \psi(\mathbb{D})$ (where $\overline{\mathbb{C}}$ stands for the Riemann sphere $\mathbb{C} \cup\{\infty\})$.

It is well known that the polynomial area theorem is equivalent to various modifications of the Grunsky and Goluzin inequalities, which are usually more important for applications than the area theorem itself. In fact, the derivation of Grunsky or Goluzin inequalities from the area theorem reduces to an effective (in an appropriate sense, say, for rational functions $p$ written in the form of sums of partial fractions) calculation of the positive and negative parts of the decomposition of $p(\psi)$ with respect to the indefinite norm (1.2).

The present paper is devoted to area theorems valid for functions $p$ that are no longer single-valued (but still analytic) in $\mathbb{C}$. More precisely, we consider functions of the form

$$
p(z)=\left(z-\mu_{1}\right)^{\theta_{1}} \cdots\left(z-\mu_{n}\right)^{\theta_{n}} q(z),
$$

where $\mu_{1}, \ldots, \mu_{n} \in \psi(\mathbb{D})$, the "branching multiplicities" $\theta_{k}$ are in the interval $(0,1)$, and $q$ is a rational function with poles in $\psi(\mathbb{D})$ (rigorously, such functions $p$ can be thought of as being defined on an appropriate subdomain of the universal covering surface of $\overline{\mathbb{C}} \backslash\left\{\mu_{1}, \ldots, \mu_{n}\right\}$ and being character-automorphic there).

In the particular case where $n=1$ and $\mu_{1}=0=\psi(0)$, area theorems of such a kind are known. Namely, if $p(z)=z^{\theta-1}$ with $\theta \in(0,1)$ and $\psi(0)=0$, then the area theorem of Prawitz 11] proved in 1927 asserts that

$$
\sum_{n \geq 0}(n+\theta)\left|c_{n}\right|^{2} \leq(1-\theta)\left|c_{-1}\right|^{2},
$$

where the coefficients $c_{n}$ are defined by

$$
p(\psi(z))=(\psi(z))^{\theta-1}=\sum_{n \geq-1} c_{n} z^{n+\theta}
$$

or (more rigorously)

$$
\left(\frac{\psi(z)}{z}\right)^{\theta-1}=\sum_{n \geq 0} c_{n-1} z^{n}
$$

A polynomial version of Prawitz' theorem was obtained by Nehari 9 ] in 1969. He proved that for $\psi(0)=0$ and $p(z)=z^{\theta-1} q(z)$ (where $q$ is regular and single-valued in $\overline{\mathbb{C}} \backslash \psi(\mathbb{D})$ ) we have

$$
\sum_{n \in \mathbb{Z}}(n+\theta)\left|c_{n}\right|^{2} \leq 0,
$$

where the coefficients $c_{n}$ are defined by the Laurent-type decomposition

$$
p(\psi(z))=\sum_{n \in \mathbb{Z}} c_{n} z^{n+\theta} .
$$

In this context, as in the case of the classical polynomial area theorem, one can effectively find a decomposition of $p(\psi)$ into positive and negative parts with respect to the indefinite norm

$$
\sum_{n \in \mathbb{Z}}(n+\theta)\left|c_{n}\right|^{2}
$$

and then derive analogs of Grunsky and Goluzin inequalities (for Grunsky-type inequalities, this was done by Nehari in the same paper [9]).

The recent paper [1 by N. Abuzyarova and H. Hedenmalm develops a geometrical approach to area theorems. Using analysis on a certain doubly connected domain, they obtained an area-type integral inequality corresponding to the case where $\psi(0)=0$, $p(z)=z^{1 / 2}(z-\psi(\lambda))^{-1 / 2}, \lambda \in \mathbb{D}$. 
In the context of general functions of the form (1.4), the following questions arise quite naturally. What is an appropriate analog of the indefinite norms (1.2) and (1.6) so that the fundamental inequality $[p(\psi), p(\psi)] \leq 0$ still survives (eventually, under some additional conditions on the function $q$ )? Is there some natural decomposition of such a norm into positive and negative parts that can be effectively calculated for functions $p(\psi)$ ? What are then appropriate analogs of the Grunsky and Goluzin inequalities?

The answer to the first of these questions is given by the well-known indefinite inner product

$$
[f, g]=\frac{i}{2 \pi} \int_{\mathbb{T}} f(\zeta) d(\overline{g(\zeta)}),
$$

where $\mathbb{T}=\partial \mathbb{D}$ is the positively oriented unit circle. The right-hand side of this formula is well defined if both $f$ and $g$ are character-automorphic (with the same character) functions defined on the universal covering of $\mathbb{T}$, because the form $f d \bar{g}$ in this case does not depend on the choice of the branch of this covering. It is easily seen that the inner products (1.2) and (1.6) are special cases of (1.7). We shall call (1.7) the Dirichlet form because for functions $f$ holomorphic in $\mathbb{D}$ the inner product $[f, f]$ reduces to the Dirichlet integral

$$
\int_{\mathbb{D}}\left|f^{\prime}(z)\right|^{2} d A(z)
$$

Here, $d A$ stands for the normalized area measure: $d A(z)=d x d y / \pi, z=x+i y$. Clearly, any function $p$ of the form (1.4) can be alternatively written as

$$
p(z)=z^{\theta_{1}+\cdots+\theta_{n}} q_{1}(z),
$$

where $q_{1}$ is regular and single-valued in a neighborhood of $\overline{\mathbb{C}} \backslash \psi(\mathbb{D})$. If $m$ is the integral part of $\theta_{1}+\cdots+\theta_{n}$, and the function $q$ has a zero of multiplicity at least $m+1$ at infinity in the case where $\theta_{1}+\cdots+\theta_{n}$ is not an integer, or of multiplicity at least $m$ in the case where $\theta_{1}+\cdots+\theta_{n}$ is an integer, then Nehari's result (or the classical polynomial area theorem) implies that

$$
[p \circ \psi, p \circ \psi] \leq 0 .
$$

Our goal in the present paper is to answer the other two of the above questions. First, it is well known from the theory of indefinite inner product spaces that the fundamental decompositions of such spaces into orthogonal sums of positive and negative subspaces are not unique (see [3, I.11]). 93 of the present paper is devoted to such decompositions with respect to the Dirichlet form, which are most relevant for functions $p(\psi)$ with $p$ of the form (1.4). Namely, in the case where $\theta_{1}+\cdots+\theta_{n} \leq 1$, we find a decomposition of the Dirichlet form (see Theorem 3.4 in 93) such that the decomposition of $p \circ \psi$ with $p$ given by (1.4) can be calculated effectively, and the rational function $q$ with poles in $\psi(\mathbb{D})$ that occurs in that formula is written as a sum of partial fractions.

This decomposition allows us to obtain corresponding area theorems. In order to formulate them in a concise form, in $\$ 4$ we introduce an abstract language of domination of kernel functions. This language gives a better understanding even of classical topics, such as the Goluzin-Lebedev inequalities for univalent functions.

Two main area theorems (Theorems 5.1 and 5.2) presented in the form of certain domination relations for kernel functions are formulated and proved in \$5. Using the domination formalism, one can interpret them either as analogs of Goluzin-Lebedev inequalities or in the form of integral estimates. Finally, $\sqrt{6}$ is devoted to examples and special cases. In the cases of one and two branching points, we obtain explicit formulas for kernel functions involved in the formulation of area theorems. 
One of the applications of our techniques is the area-type proof of the pointwise inequality:

$$
\left|\zeta \frac{F^{\prime \prime}(\zeta)}{F^{\prime}(\zeta)}+\frac{4|\zeta|^{2}-2}{|\zeta|^{2}-1}-\frac{4|\zeta|^{2}}{|\zeta|^{2}-1} \frac{E(1 /|\zeta|)}{K(1 /|\zeta|)}\right| \leq \frac{4|\zeta|^{2}}{|\zeta|^{2}-1}\left(1-\frac{E(1 /|\zeta|)}{K(1 /|\zeta|)}\right), \quad|\zeta|>1,
$$

valid for functions $F \in \Sigma$ (i.e., functions $F$ univalent in $\overline{\mathbb{C}} \backslash \overline{\mathbb{D}}$ and normalized so that $\left.F(\zeta)=\zeta+b_{0}+b_{1} \zeta^{-1}+\cdots\right)$. Here, $K$ and $E$ are standard complete elliptic integrals of the first and, respectively, second kind. This inequality was obtained by G. M. Goluzin (see [6, Chapter IV, §3]) by the variational method. Recently, N. Abuzyarova and H. Hedenmalm [1] found an integral inequality of area type leading to (1.8). Their method was based on an appropriate application of the Green formula in a certain doubly connected domain sheeted over the Riemann sphere, and elliptic functions appear in explicit formulas for the Green function of this domain. In 86 , we obtain an integral inequality equivalent to that obtained in [1] as a special case of the domination corresponding to two branching points. In our method, the elliptic integrals appear in explicit formulas for appropriate reproducing kernels.

\section{§2. Character-Automorphic Functions and the Dirichlet Form}

Let $\Omega$ be a simply connected domain in $\mathbb{C}$ and let $\mathcal{M}=\left\{\mu_{1}, \ldots, \mu_{n}\right\}$ be a finite sequence of distinct points in $\Omega$. Let $\Theta=\left\{\theta_{1}, \ldots, \theta_{n}\right\}$, where $\theta_{k} \in[0,1), k=1, \ldots, n$. We shall say that a function $f$ analytic and multivalued in $\Omega \backslash \mathcal{M}$ is $(\mathcal{M}, \Theta)$-characterautomorphic there if the analytic continuation of $f$ along any closed path $\gamma$ lying in $\Omega \backslash \mathcal{M}$ and satisfying

$$
\operatorname{ind}\left(\gamma, \mu_{k}\right)=m_{k}
$$

results in multiplication of $f$ by $\exp \left(2 \pi i\left(\theta_{1} m_{1}+\cdots+\theta_{n} m_{n}\right)\right)$. A typical example of such a function is

$$
f(z)=\left(z-\mu_{1}\right)^{\theta_{1}} \cdots\left(z-\mu_{n}\right)^{\theta_{n}}
$$

(writing such expressions, we always assume that the value of $f$ at some fixed point of the universal covering surface of $\Omega \backslash \mathcal{M}$ is specified). Moreover, if $f$ is an arbitrary $(\mathcal{M}, \Theta)$-character-automorphic function in $\Omega \backslash \mathcal{M}$, then the ratio

$$
\frac{f(z)}{\left(z-\mu_{1}\right)^{\theta_{1}} \cdots\left(z-\mu_{n}\right)^{\theta_{n}}}
$$

is $(\mathcal{M}, 0)$-character-automorphic and hence single-valued in $\Omega \backslash \mathcal{M}$. We shall say that $f$ is a meromorphic $(\mathcal{M}, \Theta)$-character-automorphic function in $\Omega$ if $f$ has the form

$$
f(z)=\left(z-\mu_{1}\right)^{\theta_{1}} \cdots\left(z-\mu_{n}\right)^{\theta_{n}} g(z),
$$

where $g$ is single-valued and meromorphic in $\Omega$. Let $\mathfrak{M}_{\mathcal{M}, \Theta}$ denote the family of all meromorphic $(\mathcal{M}, \Theta)$-character-automorphic functions in $\Omega$. Clearly, the family $\mathfrak{M}_{\mathcal{M}, \Theta}$ is linear and is closed with respect to the differentiation operator $d / d z$.

The following lemma is almost obvious.

Lemma 2.1. Suppose $\Omega$ is a simply connected domain, $\Lambda=\left\{\lambda_{1}, \ldots, \lambda_{n}\right\} \subset \Omega$, and $\psi$ is a conformal mapping from $\Omega$ onto $\Omega^{\prime}$. Also, let $\mathcal{M}=\left\{\mu_{1}, \ldots, \mu_{n}\right\}$, where $\mu_{k}=\psi\left(\lambda_{k}\right)$, $k=1, \ldots, n$. As before, $\Theta=\left\{\theta_{1}, \ldots, \theta_{n}\right\}$, with $\theta_{k} \in[0,1), k=1, \ldots, n$.

If $f$ is meromorphic $(\mathcal{M}, \Theta)$-character-automorphic in $\Omega^{\prime}$, then the composition $f \circ \psi$ is meromorphic $(\Lambda, \Theta)$-character-automorphic in $\Omega$.

More generally, let $\Omega$ be an arbitrary domain in $\mathbb{C}, \gamma$ a closed path in $\Omega$, and let $\theta \in[0,1)$. We shall say that a function $f$ analytic and multivalued in $\Omega$ is $(\gamma, \theta)$ character-automorphic if the analytic continuation of $f$ along $\gamma$ results in multiplication of $f$ by $\exp (2 \pi i \theta)$. 
In what follows, the letter $\mathbb{T}$ will denote the unit circle viewed either as a set or as a positively oriented closed path, depending on the context. We denote by $d m$ the normalized arc length measure on $\mathbb{T}$.

Let $f$ and $g$ be functions analytic (and multivalued) in some neighborhood of $\mathbb{T}$ and $(\mathbb{T}, \theta)$-character-automorphic there. The Dirichlet form $[f, g]$ is defined by

$$
[f, g]:=\frac{i}{2 \pi} \int_{\mathbb{T}} f(\zeta) d \overline{g(\zeta)} .
$$

The integral is well defined because the differential form $f d \bar{g}$ is $(\mathbb{T}, 0)$-characterautomorphic. Here are some alternative formulas for the calculation of the Dirichlet form:

$$
\begin{aligned}
{[f, g] } & =\frac{i}{2 \pi} \int_{\mathbb{T}} \partial_{\bar{z}}(f(z) \overline{g(z)}) d \bar{z} \\
& =\frac{1}{2 \pi i} \int_{\mathbb{T}} \partial_{z}(f(z) \overline{g(z)}) d z=\int_{\mathbb{T}} z \partial_{z} f(z) \overline{g(z)} d m(z)
\end{aligned}
$$

where

$$
\partial_{z}=\frac{1}{2}\left(\frac{\partial}{\partial x}-i \frac{\partial}{\partial y}\right) \quad \text { and } \quad \partial_{\bar{z}}=\frac{1}{2}\left(\frac{\partial}{\partial x}+i \frac{\partial}{\partial y}\right)
$$

are the standard Wirtinger differential operators.

Clearly, any $(\mathbb{T}, \theta)$-character-automorphic function $f$ has a representation

$$
f(z)=z^{\theta} f_{1}(z)
$$

where $f_{1}$ is analytic and single-valued near $\mathbb{T}$, whence

$$
f(z)=\sum_{k \in \mathbb{Z}} c_{k} z^{k+\theta}
$$

Writing a similar formula

$$
g(z)=\sum_{k \in \mathbb{Z}} d_{k} z^{k+\theta}
$$

for another $(\mathbb{T}, \theta)$-character automorphic function $g$, we easily obtain

$$
[f, g]=\sum_{k \in \mathbb{Z}}(k+\theta) c_{k} \overline{d_{k}} .
$$

This formula can serve as a definition of the Dirichlet form for functions $f$ and $g$ defined almost everywhere on the universal covering of $\mathbb{T}$ and having representations (2.3) and (2.4) with coefficients satisfying

$$
\sum_{k \in \mathbb{Z}}|k|\left|c_{k}\right|^{2}<+\infty \text { and } \sum_{k \in \mathbb{Z}}|k|\left|d_{k}\right|^{2}<+\infty
$$

The class of such functions will be denoted by $\mathcal{G}_{\theta}$ ( $\theta$ can be an arbitrary real number).

In the case where $\theta$ is not an integer, $\mathcal{G}_{\theta}$ supplied with the Dirichlet form is a Krĕn space. A fundamental symmetry is

$$
J f(z)=\sum_{k \in \mathbb{Z}} \operatorname{sgn}(k+\theta) c_{k} z^{k+\theta}
$$

for $f$ defined by (2.3), and the corresponding Hilbert $J$-norm is

$$
\|f\|_{J, \theta}^{2}=\sum_{k \in \mathbb{Z}}|k+\theta|\left|c_{k}\right|^{2}
$$


A natural positive (respectively, negative) subspace of $\mathcal{G}_{\theta}$ consisting of functions of the form

$$
f(z)=\sum_{k+\theta \geq 0} f_{k} z^{k+\theta}
$$

(respectively,

$$
\left.g(z)=\sum_{k+\theta \leq 0} g_{k} z^{k+\theta}\right)
$$

will be denoted by $\mathcal{G}_{\theta}^{+}$(respectively, $\mathcal{G}_{\theta}^{-}$). In the case where $\theta$ is an integer, the subspace $\{C\}$ consisting of constant functions is an isotropic subspace of $\mathcal{G}_{\theta}$, and the quotientspace $\mathcal{G}_{\theta} /\{C\}$ is a Kreı̆n space. The positive and negative subspaces $\mathcal{G}_{\theta}^{+}$and $\mathcal{G}_{\theta}^{-}$are defined as before (obviously, $\mathcal{G}_{\theta}^{+} \cap \mathcal{G}_{\theta}^{-}=\{C\}$ in the case where $\theta$ is an integer).

The formula

$$
[f, g]=\int_{\mathbb{T}} z \partial_{z} f(z) \overline{g(z)} d m(z)
$$

remains valid for arbitrary $f, g \in \mathcal{G}_{\theta}$ if $z \partial_{z} f$ is interpreted as a distribution with Fourier series

$$
h(z)=z \partial_{z} f(z) \sim \sum_{k \in \mathbb{Z}}(k+\theta) c_{k} z^{k+\theta}
$$

and the integral

$$
\int_{\mathbb{T}} h \bar{g} d m
$$

is interpreted in the sense of the Parseval identity.

From (2.5) (or from (2.2)) it follows easily that

$$
\left[z^{\eta} f(z), z^{\eta} g(z)\right]=\eta \int_{\mathbb{T}} f \bar{g} d m+[f, g]
$$

for any $f, g \in \mathcal{G}_{\theta}$. It turns out that a similar formula is true if $z^{\eta}$ is replaced by more general multivalued factors unimodular on $\mathbb{T}$.

Let $\Lambda=\left\{\lambda_{1}, \ldots, \lambda_{n}\right\} \subset \mathbb{D}$, and let $\Theta=\left\{\theta_{1}, \ldots, \theta_{n}\right\}$ with $\theta_{k} \in \mathbb{R}, k=1, \ldots, n$. We define

$$
\omega_{\Lambda, \Theta}(z):=\prod_{k=1}^{n}\left(\omega_{\lambda_{k}}(z)\right)^{\theta_{k}}
$$

where

$$
\omega_{\lambda}(z)=\frac{z-\lambda}{1-\bar{\lambda} z} .
$$

Remark. The function $\omega_{\Lambda, \Theta}$ can be thought of as a multivalued finite Blaschke product. The function

$$
\omega_{\Lambda, \mathbf{1}}(z)=\prod_{k=1}^{n} \omega_{\lambda_{k}}(z)
$$

(the usual finite Blaschke product) will be denoted simply by $\omega_{\Lambda}(z)$.

Proposition 2.1. For any $\theta \in[0,1)$ and any $f, g \in \mathcal{G}_{\theta}$, we have

$$
\left[\omega_{\Lambda, \Theta} f, \omega_{\Lambda, \Theta} g\right]=\int_{\mathbb{T}}\left(\sum_{k=1}^{n} \theta_{k} \frac{1-\left|\lambda_{k}\right|^{2}}{\left|1-\bar{\lambda}_{k} \zeta\right|^{2}}\right) f(\zeta) \overline{g(\zeta)} d m(\zeta)+[f, g] .
$$


Proof. An explicit calculation shows that

$$
\partial_{z} \omega_{\Lambda, \Theta}(z)=\left(\sum_{k=1}^{n} \theta_{k} \frac{1-\left|\lambda_{k}\right|^{2}}{\left(z-\lambda_{k}\right)\left(1-\bar{\lambda}_{k} z\right)}\right) \omega_{\Lambda, \Theta}(z)
$$

and that

$$
\frac{z\left(1-|\lambda|^{2}\right)}{(z-\lambda)(1-\bar{\lambda} z)}=\frac{1-|\lambda|^{2}}{|1-\bar{\lambda} z|^{2}}
$$

for $z \in \mathbb{T}$. Using the last identity in (2.2), we find

$$
\begin{aligned}
& {\left[\omega_{\Lambda, \Theta} f, \omega_{\Lambda, \Theta} g\right]} \\
& \quad=\int_{\mathbb{T}} z \partial_{z} \omega_{\Lambda, \Theta}(z) f(z) \overline{\omega_{\Lambda, \Theta}(z) g(z)} d m(z)+\int_{\mathbb{T}} z \omega_{\Lambda, \Theta}(z) \partial_{z} f(z) \overline{\omega_{\Lambda, \Theta}(z) g(z)} d m(z) \\
& \quad=\int_{\mathbb{T}}\left(\sum_{k=1}^{n} \theta_{k} \frac{1-\left|\lambda_{k}\right|^{2}}{\left|1-\bar{\lambda}_{k} z\right|^{2}}\right) f(z) \overline{g(z)} d m(z)+\int_{\mathbb{T}} z \partial_{z} f(z) \overline{g(z)} d m(z),
\end{aligned}
$$

which yields (2.12).

Remark. If $f=g$ is a function holomorphic in $\mathbb{D}$ and all $\theta_{k}$ are positive integers, then (2.12) is a special case of the well-known Carleson formula for the Dirichlet integral (see [4).

Now, we turn to the properties of the Dirichlet form related to different composition operators. The easiest of them is the involution $\sharp$ defined by

$$
f^{\sharp}(z):=\overline{f\left(\frac{1}{\bar{z}}\right)}
$$

for $f$ analytic near $\mathbb{T}$. Clearly, $f^{\sharp}=\bar{f}$ if we view $f$ as a function defined only on (the universal covering of $\mathbb{T}$. From (2.1), it follows immediately that

$$
\left[f^{\sharp}, g^{\sharp}\right]=-[g, f] .
$$

We also have

$$
\omega_{\Lambda, \Theta}^{\sharp}(z)=\left(\omega_{\Lambda, \Theta}(z)\right)^{-1}
$$

and

$$
\left(z \partial_{z} g\right)^{\sharp}(u)=-u \partial_{u} g^{\sharp}(u) .
$$

The following two propositions are sources of all area theorems derived in the present paper.

Proposition 2.2. Let $\Lambda=\left\{\lambda_{1}, \ldots, \lambda_{n}\right\}$ with $\lambda_{n} \in \mathbb{D}$, and let $\psi$ be a smooth conformal map of $\mathbb{D}$ into itself. Let $\psi(\Lambda)=\left\{\psi\left(\lambda_{1}\right), \ldots, \psi\left(\lambda_{n}\right)\right\}$. Suppose $p$ is a meromorphic function of class $\mathfrak{M}_{\psi(\Lambda), \Theta}$ in some domain containing the closed unit disk. Assume also that all poles of $p$ lie in the domain $\psi(\mathbb{D})$. Then

$$
[p, p]-[p \circ \psi, p \circ \psi]=\int_{\mathbb{D} \backslash \psi(\mathbb{D})}\left|p^{\prime}(z)\right|^{2} d A(z) .
$$

Proof. The left-hand side of (2.17) can be written as

$$
\frac{i}{2 \pi} \int_{\mathbb{T}} p d \bar{p}-\frac{i}{2 \pi} \int_{\mathbb{T}} p \circ \psi d \overline{p \circ \psi}=\frac{i}{2 \pi}\left(\int_{\mathbb{T}} p d \bar{p}-\int_{\psi(\mathbb{T})} p d \bar{p}\right)=\frac{i}{2 \pi} \int_{\Gamma} p d \bar{p},
$$

where $\Gamma$ is the oriented boundary of $\mathbb{D} \backslash \psi(\mathbb{D})$. Now, an application of the Green formula implies the result. 
The calculation (2.18) is by no means new. A very similar argument appears, for example, in the paper [9] (see formula (2.3) of that paper).

Formula (2.17) remains true even without any smoothness assumption on $\psi$. Indeed, any $p$ in the context of Proposition 2.2 has the form

$$
p(z)=\omega_{\psi(\Lambda), \Theta}(z)\left(\sum_{k=1}^{m} \frac{c_{k}}{\left(z-\psi\left(a_{k}\right)\right)^{\nu_{k}}}+q(z)\right)
$$

with $a_{k} \in \mathbb{D}$ and $q$ analytic in $\overline{\mathbb{D}}$. Clearly, for arbitrary $\psi$ the composition $p \circ \psi$ belongs to the class $\mathcal{G}_{\theta}$ with $\theta=\theta_{1}+\cdots+\theta_{n}$, which shows that $[p \circ \psi, p \circ \psi]$ is well defined. Moreover, the negative coefficients in the decomposition

$$
p(\psi(z))=\sum_{k \in \mathbb{Z}} c_{k} z^{k+\theta}, \quad z \in \mathbb{T}
$$

decay as

$$
\left|c_{-k}\right|=O\left(r_{0}^{k}\right), \quad k \rightarrow+\infty,
$$

for some $r_{0} \in(0,1)$. An approximation argument involving the functions $\psi_{r}(z)=\psi(r z)$, $r \in(0,1)$, now implies the claim.

Corollary 2.1. Let $\Lambda=\left\{\lambda_{1}, \ldots, \lambda_{n}\right\} \subset \mathbb{D}$, let $\psi$ be a conformal self-map of $\mathbb{D}$, and let $\Theta=\left\{\theta_{1}, \ldots, \theta_{n}\right\}, \theta_{k} \in[0,1)$. If $p$ is a meromorphic function of class $\mathfrak{M}_{\psi(\Lambda), \Theta}$ in the closed unit disk with poles in the domain $\psi(\mathbb{D})$, then

$$
[p \circ \psi, p \circ \psi] \leq[p, p],
$$

and equality occurs if and only if $\psi$ is a full self-map of $\mathbb{D}$ (i.e., $\psi$ maps $\mathbb{D}$ onto $\mathbb{D} \backslash E$, where $E$ is an area-null set).

Proposition 2.3. Let $f$ be a univalent function defined in $\mathbb{D}$. If $P$ is a function of the form

$$
P(\zeta)=\left[\prod_{k=1}^{n}\left(\zeta-f\left(\lambda_{k}\right)\right)^{\theta_{k}}\right] \sum_{\nu=1}^{n} \frac{c_{\nu}}{\zeta-f\left(u_{\nu}\right)}
$$

such that

$$
\varlimsup_{|\zeta| \rightarrow \infty}|P(\zeta)|<+\infty
$$

then

$$
-[P \circ f, P \circ f]=\int_{\mathbb{C} \backslash f(\mathbb{D})}\left|P^{\prime}(z)\right|^{2} d A(z) .
$$

Proof. First, we consider the case where $f$ is sufficiently smooth and bounded. The function $P(\zeta)$ can be written near infinity as

$$
P(\zeta)=\zeta^{\theta_{1}+\cdots+\theta_{n}} P_{1}\left(\frac{1}{\zeta}\right)
$$

where $P_{1}$ is a function regular near the origin. Condition (2.21) implies that $P_{1}$ has a zero of order at least $\theta=\theta_{1}+\cdots+\theta_{n}$ at the origin. This implies that

$$
\left|P^{\prime}(\zeta)\right|=O\left(\frac{1}{|\zeta|}\right)
$$

near infinity. We may assume without loss of generality that

$$
\lim _{|\zeta| \rightarrow \infty} P(\zeta)=0
$$

because $\varlimsup_{|\zeta| \rightarrow \infty}|P(\zeta)|>0$ is possible only if $\theta=\theta_{1}+\cdots+\theta_{n}$ is an integer and $P_{1}$ has a zero of order $\theta$ at the origin, and in this case we may replace $P(\zeta)$ by $P(\zeta)-C$, where 
$C=\lim _{|\zeta| \rightarrow \infty} P(\zeta)$, without changing $[P \circ f, P \circ f]$. Relations (2.22) and (2.23) imply that

$$
\lim _{R \rightarrow \infty} \int_{R \mathbb{T}} P d \bar{P}=0
$$

The same argument as in the proof of Proposition 2.2 shows that for sufficiently large $R$ (such that $R \mathbb{D}$ contains $f(\mathbb{D})$ ) we have

$$
\frac{i}{2 \pi} \int_{R \mathbb{T}} P d \bar{P}-[P \circ f, P \circ f]=\int_{R \mathbb{D} \backslash f(\mathbb{D})}\left|P^{\prime}(z)\right|^{2} d A(z) .
$$

It remains to let $R \rightarrow \infty$.

The case where $f$ is arbitrary can be obtained by replacing $f$ by $f_{r}(z):=f(r z)$, $r \in(0,1)$, and then letting $r \rightarrow 1-0$. Passage to the limit is justified by the following lemma.

Lemma 2.2. The function $P \circ f$ belongs to $\mathcal{G}_{\theta}, \theta=\theta_{1}+\cdots+\theta_{n}$. Moreover, the negative coefficients in the decomposition

$$
P(f(\zeta))=\sum_{k \in \mathbb{Z}} c_{k} \zeta^{k+\theta}
$$

decay as

$$
\left|c_{-k}\right|=O\left(r_{0}^{k}\right), \quad k \rightarrow+\infty,
$$

where $r_{0}$ is some number in $(0,1)$.

Proof. The lemma will follow if we show that the single-valued function

$$
Q(z)=\prod_{k=1}^{n}\left(\frac{f(\zeta)-f\left(\lambda_{k}\right)}{\zeta-\lambda_{k}}\right)^{\theta_{k}} \cdot\left(\sum_{\nu=1}^{N} \frac{c_{\nu}}{f(\zeta)-f\left(u_{\nu}\right)}\right) \cdot \prod_{\nu=1}^{N}\left(\zeta-u_{\nu}\right)
$$

belongs to $\mathcal{G}_{0}^{+}$.

Let $m$ be an integer such that $m<\theta \leq m+1$. Then condition (2.21) implies that

$$
P(\zeta)=\frac{\prod_{k=1}^{n}\left(\zeta-f\left(\lambda_{k}\right)\right)^{\theta_{k}} \cdot q(\zeta)}{\prod_{\nu=1}^{N}\left(\zeta-f\left(u_{\nu}\right)\right)},
$$

where $q$ is a polynomial of degree at most $N-m-1$. Consequently,

$$
P(\zeta)=\prod_{k=1}^{n}\left(\zeta-f\left(\lambda_{k}\right)\right)^{\theta_{k}} \sum_{\nu=m+1}^{N} \frac{d_{\nu}}{\left(\zeta-f\left(u_{\nu}\right)\right) \prod_{l=1}^{m}\left(\zeta-f\left(u_{l}\right)\right)} .
$$

We see that it suffices to show that for any choice of $u_{1}, \ldots, u_{m+1} \in \mathbb{D}$ the function

$$
R(\zeta)=\prod_{k=1}^{n}\left(\frac{f(\zeta)-f\left(\lambda_{k}\right)}{\zeta-\lambda_{k}}\right)^{\theta_{k}} \cdot \prod_{l=1}^{m+1}\left(\frac{\zeta-u_{l}}{f(\zeta)-f\left(u_{l}\right)}\right)
$$

is in $\mathcal{G}_{0}^{+}$. For this, it suffices to show that

$$
\int_{r_{0}<|\zeta|<1}\left|R^{\prime}(\zeta)\right|^{2} d A(\zeta)<+\infty
$$

for some $r_{0} \in(0,1)$. But we have

$$
\begin{aligned}
R^{\prime}(\zeta)=R(\zeta)( & \sum_{k=1}^{n} \theta_{k}\left(\frac{f^{\prime}(\zeta)}{f(\zeta)-f\left(\lambda_{k}\right)}-\frac{1}{\zeta-\lambda_{k}}\right) \\
& \left.-\sum_{l=1}^{m+1}\left(\frac{f^{\prime}(\zeta)}{f(\zeta)-f\left(u_{l}\right)}-\frac{1}{\zeta-u_{l}}\right)\right) .
\end{aligned}
$$


In the case where $\theta<m+1$, we have

$$
|R(\zeta)| \asymp \frac{1}{|f(\zeta)|^{m+1-\theta}}
$$

near $\mathbb{T}$ (we suppose without loss of generality that $f$ does not take zero values near $\mathbb{T}$ ), whence

$$
\int_{r_{0}<|\zeta|<1}\left|R^{\prime}(\zeta)\right|^{2} d A(\zeta) \asymp \int_{r_{0}<|\zeta|<1} \frac{\left|f^{\prime}(\zeta)\right|^{2}}{|f(\zeta)|^{2+2(m+1-\theta)}} d A(\zeta),
$$

which is finite by geometric reasons. In the case where $\theta=m+1$, we have

$$
\sum_{k=1}^{n} \theta_{k} \frac{f^{\prime}(\zeta)}{f(\zeta)-f\left(\lambda_{k}\right)}-\sum_{l=1}^{m+1} \frac{f^{\prime}(\zeta)}{f(\zeta)-f\left(u_{l}\right)}=O\left(\left|\frac{f^{\prime}(\zeta)}{f(\zeta)^{2}}\right|\right)
$$

near $\mathbb{T}$ due to the cancellation of first order terms as $f(\zeta) \rightarrow \infty$. Hence

$$
\left|R^{\prime}(\zeta)\right|=O\left(\left|\frac{f^{\prime}(\zeta)}{f(\zeta)^{2}}\right|\right)
$$

which gives again a finite integral (2.24).

Corollary 2.2. Let $f$ be a univalent function defined on $\mathbb{D}$. If $\theta_{1}+\cdots+\theta_{n} \leq 1$, then for any function $P$ of the form (2.20) we have

$$
[P \circ f, P \circ f] \leq 0 .
$$

Moreover, equality occurs for full mappings $f$ (i.e., those for which $\mathbb{C} \backslash f(\mathbb{D})$ is an area-null set).

\section{§3. Fundamental DeCompositions}

In this section, we study certain fundamental decompositions of the spaces $\mathcal{G}_{\theta}$ with indefinite Dirichlet form into orthogonal positive and negative subspaces.

Let $\Lambda=\left\{\lambda_{1}, \ldots, \lambda_{n}\right\}$ be a finite sequence of distinct points of $\mathbb{D}$, and $\Theta=\left\{\theta_{1}, \ldots, \theta_{n}\right\}$ a sequence of numbers $\theta_{k} \in(0,1)$. Let the function $\omega_{\Lambda, \Theta}$ be defined by (2.9) and (2.10). An easy argument (which is left to the reader) shows that multiplication by $\omega_{\Lambda, \Theta}$ is an invertible transformation from $\mathcal{G}_{\eta}$ onto $\mathcal{G}_{\nu}$, where

$$
\nu=\eta+\theta_{1}+\cdots+\theta_{n}
$$

In the case where both $\eta$ and $\nu$ are not integers, this multiplication operator is continuous and invertible as an operator between Kreĭn spaces.

Definition 3.1. Let $\theta=\theta_{1}+\cdots+\theta_{n}$. A subspace of $\mathcal{G}_{\theta}$ consisting of functions $f$ of the form

will be denoted by $\mathcal{G}_{\Lambda, \Theta}^{+}$.

$$
f=\omega_{\Lambda, \Theta} g \quad \text { with } \quad g \in \mathcal{G}_{0}^{+}
$$

Applying (2.12), we see that

$$
\begin{aligned}
{\left[\omega_{\Lambda, \Theta} g, \omega_{\Lambda, \Theta} g\right] } & =\int_{\mathbb{T}}\left(\sum_{k=1}^{n} \theta_{k} \frac{1-\left|\lambda_{k}\right|^{2}}{\left|1-\bar{\lambda}_{k} \zeta\right|^{2}}\right)|g(\zeta)|^{2} d m(\zeta)+[g, g] \\
& =\int_{\mathbb{T}}\left(\sum_{k=1}^{n} \theta_{k} \frac{1-\left|\lambda_{k}\right|^{2}}{\left|1-\bar{\lambda}_{k} \zeta\right|^{2}}\right)|g(\zeta)|^{2} d m(\zeta)+\sum_{k \geq 1} k|\hat{g}(k)|^{2}
\end{aligned}
$$

for any $g \in \mathcal{G}_{0}^{+}$, which shows that $\mathcal{G}_{\Lambda, \Theta}^{+}$is a positive subspace of $\mathcal{G}_{\theta}$. Moreover, it is also uniformly positive as a subspace of a Kreĭn space provided $\theta \notin \mathbb{Z}$, which means that

$$
[f, f] \geq c\|f\|_{J, \theta}^{2}
$$


for any $f \in \mathcal{G}_{\Lambda, \Theta}^{+}$. Later on in this section, we shall prove that $\mathcal{G}_{\Lambda, \Theta}^{+}$is a maximal positive subspace (which means that it is not contained in any larger positive subspace) in the case where $\theta_{1}+\cdots+\theta_{n}<1$. Regarded as a space of functions analytic in $\mathbb{D}$, the Dirichlet space $\mathcal{G}_{0}^{+}$supplied with the norm

$$
\|g\|_{\mathcal{D}_{\Lambda, \Theta}}^{2}:=\int_{\mathbb{T}}\left(\sum_{k=1}^{n} \theta_{k} \frac{1-\left|\lambda_{k}\right|^{2}}{\left|1-\bar{\lambda}_{k} \zeta\right|^{2}}\right)|g(\zeta)|^{2} d m(\zeta)+\sum_{k \geq 1} k|\hat{g}(k)|^{2}=\left[\omega_{\Lambda, \Theta} g, \omega_{\Lambda, \Theta} g\right]
$$

will be denoted by $\mathcal{D}_{\Lambda, \Theta}$. Let $k_{\Lambda, \Theta}^{+}$denote the reproducing kernel for the space $\mathcal{D}_{\Lambda, \Theta}$. In other words, for each $\mu \in \mathbb{D}$, the function $k_{\Lambda, \Theta}^{+}(\cdot, \mu) \in \mathcal{D}_{\Lambda, \Theta}$ is uniquely determined by the identity

$$
g(\mu)=\left[\omega_{\Lambda, \Theta} g, \omega_{\Lambda, \Theta} k_{\Lambda, \Theta}^{+}(\cdot, \mu)\right], \quad g \in \mathcal{G}_{0}^{+} .
$$

The following lemma gives an integral representation of the norm in the space $\mathcal{D}_{\Lambda, \Theta}$.

Lemma 3.1. For any $g \in \mathcal{D}_{\Lambda, \Theta}$ we have

$$
\|g\|_{\mathcal{D}_{\Lambda, \Theta}}^{2}=\int_{\mathbb{D}}\left|\left(\sum_{k=1}^{n} \theta_{k} \frac{1-\left|\lambda_{k}\right|^{2}}{\left(1-\bar{\lambda}_{k} z\right)^{2}} \omega_{\Lambda}^{k}(z)\right) g(z)+\omega_{\Lambda}(z) g^{\prime}(z)\right|^{2}\left|\omega_{\Lambda, \Theta-\mathbf{1}}(z)\right|^{2} d A(z) .
$$

Here, $\omega_{\Lambda}$ is defined by (2.11),

$$
\omega_{\Lambda}^{k}(z)=\frac{\omega_{\Lambda}(z)}{\omega_{\lambda_{k}}(z)}
$$

and $\Theta-1$ means the sequence $\left(\theta_{1}-1, \ldots, \theta_{n}-1\right)$.

Proof. Let $\mathbb{D}_{\Lambda, \varepsilon}$ be the unit disk with deleted disks of small radius $\varepsilon>0$ around the points $\lambda_{k}, k=1, \ldots, n$. Then

$$
\begin{aligned}
\|g\|_{\Lambda, \Theta}^{2} & =\left[\omega_{\Lambda, \Theta} g, \omega_{\Lambda, \Theta} g\right]=\lim _{\varepsilon \rightarrow 0} \frac{i}{2 \pi} \int_{\partial \mathbb{D}_{\Lambda, \varepsilon}} \omega_{\Lambda, \Theta}(z) g(z) d\left(\overline{\omega_{\Lambda, \Theta}(z) g(z)}\right) \\
& =\lim _{\varepsilon \rightarrow 0} \int_{\mathbb{D}_{\Lambda, \varepsilon}}\left|\partial_{z}\left(\omega_{\Lambda, \Theta}(z) g(z)\right)\right|^{2} d A(z) \\
& =\int_{\mathbb{D}}\left|\left(\sum_{k=1}^{n} \theta_{k} \frac{1-\left|\lambda_{k}\right|^{2}}{\left(z-\lambda_{k}\right)\left(1-\bar{\lambda}_{k} z\right)}\right) \omega_{\Lambda, \Theta}(z) g(z)+\omega_{\Lambda, \Theta}(z) g^{\prime}(z)\right|^{2} d A(z),
\end{aligned}
$$

which gives (3.3).

Our next goal is a characterization and the study of the orthogonal companion to $\mathcal{G}_{\Lambda, \Theta}^{+}$ with respect to the Dirichlet form. Let $\theta=\theta_{1}+\cdots+\theta_{n}$.

Definition 3.2. A subspace of $\mathcal{G}_{-\theta}$ consisting of functions $H$ satisfying

$$
\left[H^{\sharp}, g\right]=0
$$

for any $g \in \mathcal{G}_{\Lambda, \Theta}^{+}$will be denoted by $\mathcal{G}_{\Lambda, \Theta}^{-}$.

In other words, $\left(\mathcal{G}_{\Lambda, \Theta}^{-}\right)^{\sharp}$ is the orthogonal companion to $\mathcal{G}_{\Lambda, \Theta}^{+}$.

Let $\mathcal{B}_{0}^{+}$denote the usual Bergman space of single-valued functions $x$ analytic in $\mathbb{D}$ and satisfying

$$
\|x\|_{\mathcal{B}_{0}^{+}}^{2}=\int_{\mathbb{D}}|x(z)|^{2} d A(z)=\sum_{n \geq 0} \frac{|\hat{x}(n)|^{2}}{n+1}<+\infty .
$$

We shall need also a two-sided character-automorphic Bergman space $\mathcal{B}_{\theta}$ consisting of distributions $h$ defined on the universal covering of $\mathbb{T}$, having Fourier series

$$
h(u) \sim \sum_{k \in \mathbb{Z}} h_{k} u^{k+\theta},
$$


and satisfying

$$
\sum_{k \in \mathbb{Z}} \frac{\left|h_{k}\right|^{2}}{|k|+1}<+\infty
$$

Obviously, $z \partial_{z} f \in \mathcal{B}_{\theta}$ if and only if $f \in \mathcal{G}_{\theta}$. Multiplication by $\omega_{\Lambda, \Theta}$ is an invertible transformation from $\mathcal{B}_{\eta}$ onto $\mathcal{B}_{\nu}$, where $\nu=\eta+\theta_{1}+\cdots+\theta_{n}$ (the proof of this fact is left to the reader).

Lemma 3.2. A function $H \in \mathcal{G}_{-\theta}$ belongs to $\mathcal{G}_{\Lambda, \Theta}^{-}$if and only if

$$
u \partial_{u} H(u)=u \omega_{\Lambda, \Theta}{ }^{-1}(u) x(u)
$$

on (the universal covering of) $\mathbb{T}$, where $x$ is a function in $\mathcal{B}_{0}^{+}$.

Proof. By definition, the fact that $H \in \mathcal{G}_{\Lambda, \Theta}^{-}$is equivalent to the identity

$$
\left[H^{\sharp}, \omega_{\Lambda, \Theta} p\right]=0
$$

for any analytic polynomial $p$, which, by (2.7) and (2.16), can be rewritten as

$$
\begin{aligned}
0 & =\int_{\mathbb{T}} z \partial_{z} H^{\sharp}(z) \overline{\omega_{\Lambda, \Theta}(z) p(z)} d m(z) \\
& =-\int_{\mathbb{T}}\left(u \partial_{u} H\right)^{\sharp}(u) \overline{\omega_{\Lambda, \Theta}(u) p(u)} d m(u) \\
& =-\int_{\mathbb{T}} \omega_{\Lambda, \Theta}(u) u \partial_{u} H(u) p(u) d m(u) .
\end{aligned}
$$

Now the assertion is obvious.

A natural question in connection with this lemma is which functions $x$ can appear in (3.4). The answer depends on whether $\theta=\theta_{1}+\cdots+\theta_{n}$ is an integer or not.

If $\theta$ is an integer, then $\omega_{\Lambda, \Theta}{ }^{-1} x \in \mathcal{B}_{0}$ for $x \in \mathcal{B}_{0}^{+}$, and the existence of $H \in \mathcal{G}_{0}$ satisfying (3.4) is equivalent to the property

$$
\int_{\mathbb{T}} u \omega_{\Lambda, \Theta}^{-1}(u) x(u) d m(u)=0
$$

or

$$
\int_{\gamma} \frac{x(u)}{\omega_{\Lambda, \Theta}(u)} d u=0
$$

where $\gamma$ is an arbitrary simple contour in $\mathbb{D}$ enclosing the points $\lambda_{1}, \ldots, \lambda_{n}$. In other words, the function $\omega_{\Lambda, \Theta}{ }^{-1}(u) x(u)$, which is analytic and single-valued in some annulus $\{r<|u|<1\}$, where $\left|\lambda_{k}\right|<r, k=1, \ldots, n$, should have a single-valued primitive function in this annulus.

In the case where $\theta$ is not an integer, formula (2.8) shows that any function $x \in \mathcal{B}_{0}^{+}$ can appear in (3.4).

The next question is how to express the Dirichlet form $[H, H]$ in terms of the function $x$ if $H$ satisfies (3.4). The difficulty here is that $H$ satisfying (3.4) need not be a boundary value of a $(\Lambda,-\theta)$-character-automorphic function, so that one cannot apply Green's formula to the domain $\mathbb{D} \backslash\left\{\lambda_{1}, \ldots, \lambda_{n}\right\}$ as we did in the proof of Lemma 3.1 .

First, we consider the case where $\theta$ is not an integer. So, we assume that $H \in \mathcal{G}_{\Lambda, \Theta}^{-}$ and

$$
\partial_{u} H(u)=h(u)=\frac{x(u)}{\omega_{\Lambda, \Theta}(u)}
$$


on (the universal covering of) $\mathbb{T}$, where $x \in \mathcal{B}_{0}^{+}$. Obviously, the right-hand side is a $(\Lambda,-\theta)$-character-automorphic function in $\mathbb{D} \backslash\left\{\lambda_{1}, \ldots, \lambda_{n}\right\}$. We choose some simple smooth paths $\gamma_{1}, \ldots, \gamma_{n-1}$ such that

- $\gamma_{k}$ connects $\lambda_{n}$ with $\lambda_{k}$;

- $\gamma_{i}$ intersects $\gamma_{j}$ only at the point $\lambda_{n}$, and

- $\varphi_{k}=\arg \gamma_{k}^{\prime}$ at the point $\lambda_{n}$ can be fixed so that

$$
\varphi^{*}<\varphi_{1}<\varphi_{2}<\cdots<\varphi_{n-1}<\varphi^{*}+2 \pi
$$

where $\varphi^{*}$ is some value (see Figure 1). A path $\gamma$ in $\mathbb{D} \backslash\left\{\lambda_{1}, \ldots, \lambda_{n}\right\}$ is then constructed as follows. A starting point $\lambda_{n}^{0}$ is chosen on the path $\gamma_{1}$ infinitesimally close to $\lambda_{n}$. Then we go from $\lambda_{n}^{0}$ to $\lambda_{1}$ along $\gamma_{1}$, obtaining a path $\gamma_{1}^{-}$; then we go around $\lambda_{1}$ along an infinitesimally small circle in the positive direction; then back to $\lambda_{n}$ along $\gamma_{1}$, obtaining a path $\gamma_{1}^{+}$; then to the path $\gamma_{2}$ along an arc of an infinitesimally small circle centered at $\lambda_{n}$ in the positive direction; then along $\gamma_{2}$ to $\lambda_{2}$, obtaining a path $\gamma_{2}^{-}$, around $\lambda_{2}$ and back to $\lambda_{n}$ along $\gamma_{2}$, obtaining $\gamma_{2}^{+}$, and so on, returning finally to $\lambda_{n}^{0}$ along an arc of an infinitesimally small circle centered at $\lambda_{n}$ in the positive direction (see Figure 1). On the universal covering of $\mathbb{D} \backslash\left\{\lambda_{1}, \ldots, \lambda_{n}\right\}$ we fix some point $\lambda_{n}^{*}$ whose projection to $\mathbb{D}$ is $\lambda_{n}^{0}$. The lifting of $\gamma$ to the universal covering of $\mathbb{D} \backslash\left\{\lambda_{1}, \ldots, \lambda_{n}\right\}$ will be denoted by $\gamma^{*}$. Also, let $\Omega \subset \mathbb{D}$ be some smooth simply connected domain containing all paths $\gamma_{k}$ except the endpoints $\lambda_{j}, j=1, \ldots, n$. Let $h_{0}$ be a single-valued branch of the function $h$ defined on $\Omega$ and satisfying $h_{0}\left(\lambda_{n}^{0}\right)=h\left(\lambda_{n}^{*}\right)$. We put

$$
I_{k}:=\int_{\gamma_{k}^{-}} h_{0}(u) d u, \quad k=1, \ldots, n-1 .
$$

The path $\gamma$ constructed above is homotopically equivalent to $\mathbb{T}$ in $\mathbb{D} \backslash\left\{\lambda_{1}, \ldots, \lambda_{n}\right\}$. If a simply-connected domain $\Omega \subset \mathbb{D}$ containing the points $\lambda_{1}, \ldots, \lambda_{n}$ on its boundary is already given, then the existence of the paths $\gamma_{1}, \ldots, \gamma_{n-1}$ lying in $\Omega$ and satisfying the above requirements is equivalent to the property that the sequence $\lambda_{1}, \ldots, \lambda_{n}$ follows the positive orientation of $\partial \Omega$.

Lemma 3.3. There exists a unique function $H$ defined and multivalued in the domain $\mathbb{D} \backslash\left\{\lambda_{1}, \ldots, \lambda_{n}\right\}$, satisfying (3.6), and $(\Gamma,-\theta)$-character-automorphic for any simple positively oriented path $\Gamma$ in $\mathbb{D}$ enclosing the points $\left\{\lambda_{1}, \ldots, \lambda_{n}\right\}$. Moreover, this function $H$ satisfies

$$
H\left(\lambda_{n}^{*}\right)=\left(e^{-2 \pi i \theta}-1\right)^{-1} \cdot \sum_{k=1}^{n-1}\left(1-e^{-2 \pi i \theta_{k}}\right) e^{-2 \pi i\left(\theta_{1}+\cdots+\theta_{k-1}\right)} I_{k} .
$$

Proof. Let $H$ be some function satisfying (3.6), and let $H\left(\lambda_{n}^{*}\right)=C$. First, we show that this constant $C$ (and hence the function $H$ ) is uniquely determined by the requirement that $H$ be $(\gamma,-\theta)$-character-automorphic. The process of analytic continuation of the function $h$ along the path $\gamma^{*}$ shows that

$$
\begin{array}{ll}
h(u)=h_{0}(u) \text { on } \gamma_{1}^{-} ; & h(u)=e^{-2 \pi i \theta_{1}} h_{0}(u) \text { on } \gamma_{1}^{+} ; \\
h(u)=e^{-2 \pi i \theta_{1}} h_{0}(u) \text { on } \gamma_{2}^{-} ; & h(u)=e^{-2 \pi i\left(\theta_{1}+\theta_{2}\right)} h_{0}(u) \text { on } \gamma_{2}^{+} ;
\end{array}
$$




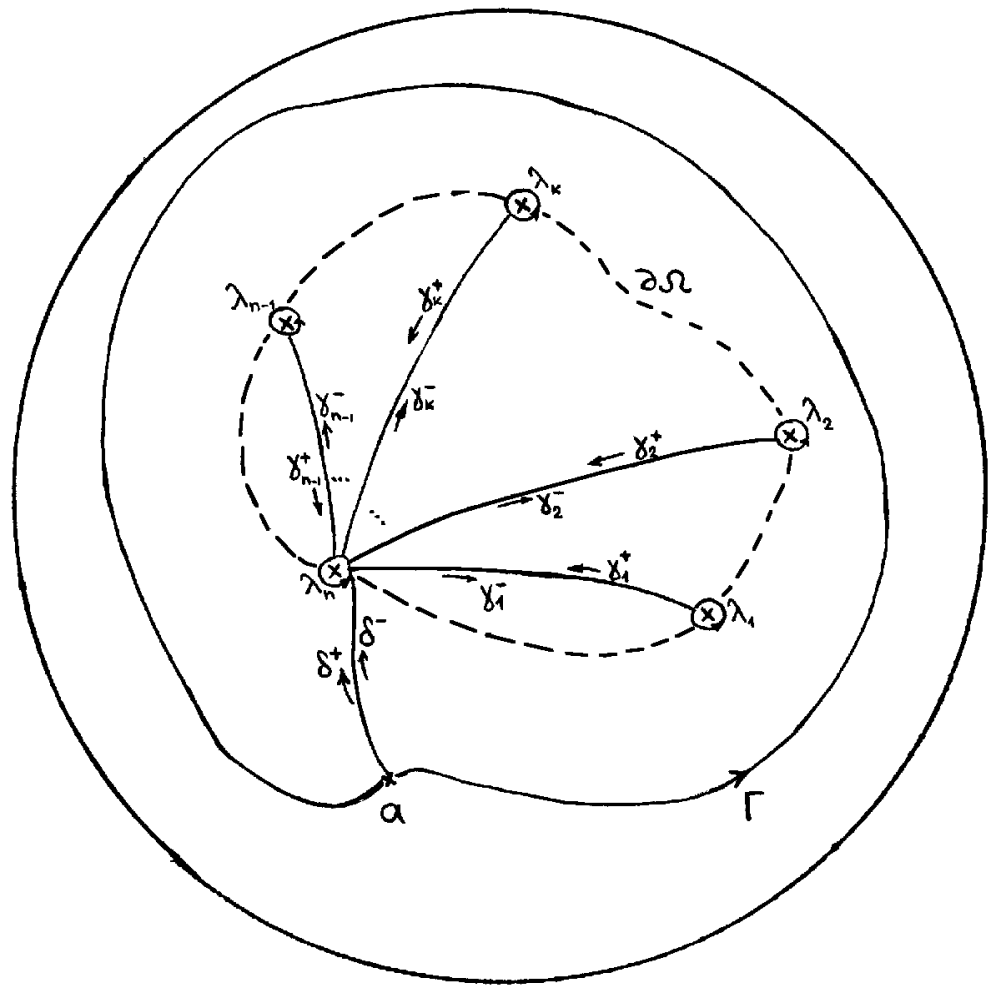

Figure 1.

and so on. Hence,

$$
\begin{array}{rl}
\int_{\gamma^{*}} & h(u) d u=\sum_{k=1}^{n-1}\left(\int_{\gamma_{k}^{-}}+\int_{\gamma_{k}^{+}}\right) h(u) d u \\
= & \sum_{k=1}^{n-1}\left(\int_{\gamma_{k}^{-}} e^{-2 \pi i\left(\theta_{1}+\cdots+\theta_{k-1}\right)} h_{0}(u) d u-\int_{\gamma_{k}^{-}} e^{-2 \pi i\left(\theta_{1}+\cdots+\theta_{k}\right)} h_{0}(u) d u\right) \\
= & \sum_{k=1}^{n-1} \int_{\gamma_{\bar{k}}^{-}}\left(1-e^{-2 \pi i \theta_{k}}\right) e^{-2 \pi i\left(\theta_{1}+\cdots+\theta_{k-1}\right)} h_{0}(u) d u \\
= & \sum_{k=1}^{n-1}\left(1-e^{-2 \pi i \theta_{k}}\right) e^{-2 \pi i\left(\theta_{1}+\cdots+\theta_{k-1}\right)} I_{k} .
\end{array}
$$

The requirement that $H$ be $(\gamma,-\theta)$-character-automorphic implies that

$$
H\left(\lambda_{n}^{*}\right)+\int_{\gamma^{*}} h(u) d u=e^{-2 \pi i \theta} H\left(\lambda_{n}^{*}\right),
$$

which gives

$$
C=\left(e^{-2 \pi i \theta}-1\right)^{-1} \sum_{k=1}^{n-1}\left(1-e^{-2 \pi i \theta_{k}}\right) e^{-2 \pi i\left(\theta_{1}+\cdots+\theta_{k-1}\right)} I_{k}
$$

and proves (3.8). 
It remains to show that if $H$ is the function primitive to $h$ and satisfying $H\left(\lambda_{n}^{*}\right)=C$, where $C$ is given by (3.9), then $H$ is $(\Gamma,-\theta)$-character-automorphic for any simple positively oriented path $\Gamma$ enclosing the points $\left\{\lambda_{1}, \ldots, \lambda_{n}\right\}$ (i.e., homotopically equivalent to $\mathbb{T}$ in $\left.\mathbb{D} \backslash\left\{\lambda_{1}, \ldots, \lambda_{n}\right\}\right)$. Let $\Gamma^{*}$ denote the lifting of such a path to the universal covering of $\mathbb{D} \backslash\left\{\lambda_{1}, \ldots, \lambda_{n}\right\}$ with the initial point $a^{-}$and the endpoint $a^{+}$. Also, suppose $\delta^{-}$is an arbitrary path on the universal covering connecting $a^{-}$and $\lambda_{n}^{*}$, and $\left(\delta^{+}\right)^{-1}$ is the image of $\delta^{-}$under the canonical isomorphism of the universal covering induced by the homotopy class of paths $\tau$ equivalent to $\mathbb{T}$, so that, in particular, the endpoint of $\delta^{+}$is $a^{+}$(see Figure 1). Since $\gamma$ and $\Gamma$ are both homotopically equivalent to $\mathbb{T}$, we have

$$
\int_{\Gamma^{*}} h(u) d u=\int_{\delta^{-}} h(u) d u+\int_{\gamma^{*}} h(u) d u+\int_{\delta^{+}} h(u) d u,
$$

which implies

$$
\begin{aligned}
H\left(a^{+}\right) & =H\left(a^{-}\right)+\int_{\delta^{-}} h(u) d u+\left(e^{-2 \pi i \theta}-1\right) H\left(\lambda_{n}^{*}\right)-e^{-2 \pi i \theta} \int_{\delta^{-}} h(u) d u \\
& =H\left(a^{-}\right)+\left(e^{-2 \pi i \theta}-1\right)\left(H\left(\lambda_{n}^{*}\right)-\int_{\delta^{-}} h(u) d u\right) \\
& =H\left(a^{-}\right)+\left(e^{-2 \pi i \theta}-1\right) H\left(a^{-}\right)=e^{-2 \pi i \theta} H\left(a^{-}\right) .
\end{aligned}
$$

This proves that $H$ is $(\Gamma,-\theta)$-character-automorphic.

Now, we apply Green's formula to the domain $\mathbb{D} \backslash \operatorname{supp} \gamma$, obtaining

$$
\begin{aligned}
{[H, H] } & =\int_{\mathbb{T}} \partial_{u} H(u) \cdot \overline{H(u)} \frac{d u}{2 \pi i} \\
& =\int_{\gamma} h(u) \overline{H(u)} \frac{d u}{2 \pi i}+\int_{\mathbb{D} \backslash \operatorname{supp} \gamma}\left|\partial_{u} H(u)\right|^{2} d A(u) \\
& =\int_{\gamma} h(u) \overline{H(u)} \frac{d u}{2 \pi i}+\int_{\mathbb{D}}|x(u)|^{2} \frac{d A(u)}{\left|\omega_{\Lambda, \Theta}(u)\right|^{2}} .
\end{aligned}
$$

The first term in this formula can be expressed via the integrals $I_{k}$. First, we have

$$
H\left(u^{-}\right)=C+\int_{\lambda_{n}^{*}}^{u^{-}} h(v) d v=C+\int_{\lambda_{n}}^{u^{-}} h_{0}(v) d v \quad \text { on the path } \gamma_{1}^{-} ;
$$

here $C=H\left(\lambda_{n}^{*}\right)$ is given by (3.9), and the last integral is taken along $\gamma_{1}$. Since $h(v)=$ $e^{-2 \pi i \theta_{1}} h_{0}(v)$ along $\gamma_{1}^{+}$, we see that

$$
\begin{aligned}
H\left(u^{+}\right) & =C+I_{1}+\int_{\lambda_{1}}^{u^{+}} e^{-2 \pi i \theta_{1}} h_{0}(v) d v \\
& =C+\left(1-e^{-2 \pi i \theta_{1}}\right) I_{1}+e^{-2 \pi i \theta_{1}} \int_{\lambda_{n}}^{u^{+}} h_{0}(v) d v
\end{aligned}
$$

on the path $\gamma_{1}^{+}$, the last integral being taken along $\gamma_{1}$. Continuing similarly, we find

$$
H\left(u^{-}\right)=C+\sum_{l=1}^{k-1}\left(1-e^{-2 \pi i \theta_{l}}\right) e^{-2 \pi i\left(\theta_{1}+\cdots+\theta_{l-1}\right)} I_{l}+e^{-2 \pi i\left(\theta_{1}+\cdots+\theta_{k-1}\right)} \int_{\lambda_{n}}^{u^{-}} h_{0}(v) d v
$$

on the path $\gamma_{k}^{-}$and

$$
H\left(u^{+}\right)=C+\sum_{l=1}^{k}\left(1-e^{-2 \pi i \theta_{l}}\right) e^{-2 \pi i\left(\theta_{1}+\cdots+\theta_{l-1}\right)} I_{l}+e^{-2 \pi i\left(\theta_{1}+\cdots+\theta_{k}\right)} \int_{\lambda_{n}}^{u^{+}} h_{0}(v) d v
$$


on the path $\gamma_{k}^{+}$, both integrals in these formulas being taken along $\gamma_{k}$. In particular,

$$
H\left(u^{+}\right)-H\left(u^{-}\right)=e^{-2 \pi i\left(\theta_{1}+\cdots+\theta_{k-1}\right)}\left(1-e^{-2 \pi i \theta_{k}}\right) \int_{u}^{\lambda_{k}} h_{0}(v) d v
$$

if $u^{-}, u^{+}$denote some points $u \in \operatorname{supp} \gamma_{k}$ interpreted as lying on $\gamma_{k}^{-}, \gamma_{k}^{+}$, respectively. Now, we have

$$
\begin{aligned}
\left(\int_{\gamma_{k}^{-}}+\int_{\gamma_{k}^{+}}\right) h(u) \overline{H(u)} \frac{d u}{2 \pi i}=\int_{\lambda_{n}}^{\lambda_{k}}\left(h\left(u^{-}\right) \overline{H\left(u^{-}\right)}-h\left(u^{+}\right) \overline{H\left(u^{+}\right)}\right) \frac{d u}{2 \pi i} \\
=\int_{\lambda_{n}}^{\lambda_{k}}\left(h\left(u^{-}\right) \overline{H\left(u^{-}\right)}-h\left(u^{+}\right) \overline{H\left(u^{-}\right)}\right. \\
\quad-h\left(u^{+}\right) e^{2 \pi i\left(\theta_{1}+\cdots+\theta_{k-1}\right)}\left(1-e^{2 \pi i \theta_{k}}\right) \overline{\left.\int_{u}^{\lambda_{k}} h_{0}(v) d v\right)} \frac{d u}{2 \pi i} \\
=\int_{\gamma_{k}^{-}}\left(\left(1-e^{-2 \pi i \theta_{k}}\right) h\left(u^{-}\right) \overline{H\left(u^{-}\right)}\right. \\
\left.\quad-h\left(u^{-}\right) e^{2 \pi i\left(\theta_{1}+\cdots+\theta_{k-1}\right)}\left(e^{-2 \pi i \theta_{k}}-1\right) \overline{\int_{u}^{\lambda_{k}} h_{0}(v) d v}\right) \frac{d u}{2 \pi i} .
\end{aligned}
$$

Using the identity

$$
\begin{aligned}
H\left(u^{-}\right) & +e^{-2 \pi i\left(\theta_{1}+\cdots+\theta_{k-1}\right)} \int_{u}^{\lambda_{k}} h_{0}(v) d v \\
& =C+\sum_{l=1}^{k-1}\left(1-e^{-2 \pi i \theta_{l}}\right) e^{-2 \pi i\left(\theta_{1}+\cdots+\theta_{l-1}\right)} I_{l}+e^{-2 \pi i\left(\theta_{1}+\cdots+\theta_{k-1}\right)} I_{k},
\end{aligned}
$$

we see that the last expression in (3.15) reduces to

$$
\begin{aligned}
\left(1-e^{-2 \pi i \theta_{k}}\right) \int_{\gamma_{k}^{-}} h\left(u^{-}\right) \\
\quad \times\left(\bar{C}+\sum_{l=1}^{k-1}\left(1-e^{2 \pi i \theta_{l}}\right) e^{2 \pi i\left(\theta_{1}+\cdots+\theta_{l-1}\right)} \overline{I_{l}}+e^{2 \pi i\left(\theta_{1}+\cdots+\theta_{k-1}\right)} \overline{I_{k}}\right) \frac{d u}{2 \pi i} \\
=\frac{1}{2 \pi i}\left(1-e^{-2 \pi i \theta_{k}}\right) e^{-2 \pi i\left(\theta_{1}+\cdots+\theta_{k-1}\right)} I_{k} \\
\quad \times\left(\bar{C}+\sum_{l=1}^{k-1}\left(1-e^{2 \pi i \theta_{l}}\right) e^{2 \pi i\left(\theta_{1}+\cdots+\theta_{l-1}\right)} \overline{I_{l}}+e^{2 \pi i\left(\theta_{1}+\cdots+\theta_{k-1}\right)} \overline{I_{k}}\right) .
\end{aligned}
$$

Using the identity

$$
\begin{aligned}
C+ & \sum_{l=1}^{k-1}\left(1-e^{-2 \pi i \theta_{l}}\right) e^{-2 \pi i\left(\theta_{1}+\cdots+\theta_{l-1}\right)} I_{l}+e^{-2 \pi i\left(\theta_{1}+\cdots+\theta_{k-1}\right)} I_{k} \\
= & \sum_{l=1}^{k-1} e^{-2 \pi i \theta} \frac{\left(1-e^{-2 \pi i \theta_{l}}\right) e^{-2 \pi i\left(\theta_{1}+\cdots+\theta_{l-1}\right)}}{e^{-2 \pi i \theta}-1} I_{l} \\
& +\frac{e^{-2 \pi i \theta}-e^{-2 \pi i \theta_{k}}}{e^{-2 \pi i \theta}-1} \cdot e^{-2 \pi i\left(\theta_{1}+\cdots+\theta_{k-1}\right)} I_{k} \\
& +\sum_{l=k+1}^{n-1} \frac{\left(1-e^{-2 \pi i \theta_{l}}\right) e^{-2 \pi i\left(\theta_{1}+\cdots+\theta_{l-1}\right)}}{e^{-2 \pi i \theta}-1} I_{l},
\end{aligned}
$$


we find

$$
\begin{aligned}
& \left(\int_{\gamma_{k}^{-}}+\int_{\gamma_{k}^{+}}\right) h(u) \overline{H(u)} \frac{d u}{2 \pi i} \\
& =\frac{1}{2 \pi i} \sum_{l=1}^{k-1}\left(1-e^{-2 \pi i \theta_{k}}\right) \overline{\left(1-e^{-2 \pi i \theta_{l}}\right)} e^{-2 \pi i\left(\theta_{1}+\cdots+\theta_{k-1}\right)} e^{2 \pi i\left(\theta_{1}+\cdots+\theta_{l-1}\right)} \frac{e^{2 \pi i \theta}}{e^{2 \pi i \theta}-1} I_{k} \overline{I_{l}} \\
& \quad+\frac{1}{2 \pi i} \frac{\left(1-e^{-2 \pi i \theta_{k}}\right)\left(e^{2 \pi i \theta}-e^{2 \pi i \theta_{k}}\right)}{e^{2 \pi i \theta}-1}\left|I_{k}\right|^{2} \\
& \quad+\frac{1}{2 \pi i} \sum_{l=k+1}^{n-1}\left(1-e^{-2 \pi i \theta_{k}}\right) \overline{\left(1-e^{-2 \pi i \theta_{l}}\right)} e^{-2 \pi i\left(\theta_{1}+\cdots+\theta_{k-1}\right)} e^{2 \pi i\left(\theta_{1}+\cdots+\theta_{l-1}\right)} \frac{1}{e^{2 \pi i \theta}-1} I_{k} \overline{I_{l}} .
\end{aligned}
$$

If we denote

$$
J_{k}:=\left(1-e^{-2 \pi i \theta_{k}}\right) e^{-2 \pi i\left(\theta_{1}+\cdots+\theta_{k-1}\right)} I_{k}
$$

then the last expression reduces to

$$
\frac{1}{4 \pi \sin \pi \theta}\left(-\sum_{l=1}^{k-1} e^{\pi i \theta} J_{k} \overline{J_{l}}+\frac{\sin \pi\left(\theta-\theta_{k}\right)}{\sin \pi \theta_{k}}\left|J_{k}\right|^{2}-\sum_{l=k+1}^{n-1} e^{-\pi i \theta} J_{k} \overline{J_{l}}\right) .
$$

This implies the identity

$$
\int_{\gamma} h(u) \overline{H(u)} \frac{d u}{2 \pi i}=\frac{1}{4 \pi \sin \pi \theta} \mathbf{Q}_{\Theta}\left(J_{1}, \ldots, J_{n-1}\right),
$$

where the quadratic form $\mathbf{Q}_{\Theta}$ is given by the formula

$$
\mathbf{Q}_{\Theta}\left(J_{1}, \ldots, J_{n-1}\right):=\sum_{k=1}^{n-1} \frac{\sin \pi\left(\theta-\theta_{k}\right)}{\sin \pi \theta_{k}}\left|J_{k}\right|^{2}-2 \operatorname{Re}\left(\sum_{1 \leq l \leq k-1} e^{\pi i \theta} J_{k} \overline{J_{l}}\right) .
$$

Finally, we arrive at the following statement.

Theorem 3.1. Assume that $\theta=\theta_{1}+\cdots+\theta_{n}$ is not an integer. Then $H \in \mathcal{G}_{\Lambda, \Theta}^{-}$if and only if there exists a function $x \in \mathcal{B}_{0}^{+}$such that

$$
\partial_{u} H(u)=h(u)=\frac{x(u)}{\omega_{\Lambda, \Theta}(u)} .
$$

Moreover, let $\Omega \subset \mathbb{D}$ be a smooth simply connected domain such that the sequence $\left\{\lambda_{1}, \ldots, \lambda_{n}\right\}$ lies on the boundary $\partial \Omega$ and follows the positive orientation of $\partial \Omega$. Let $h_{0}$ be some single-valued branch of $h$ defined in $\Omega$, and let

$$
J_{k}=\left(1-e^{-2 \pi i \theta_{k}}\right) e^{-2 \pi i\left(\theta_{1}+\cdots+\theta_{k-1}\right)} \int_{\lambda_{n}}^{\lambda_{k}} h_{0}(u) d u, \quad k=1, \ldots, n-1
$$

(the integral is taken along some path lying in $\Omega$ ). Then

$$
[H, H]=\int_{\mathbb{D}}|x(u)|^{2} \frac{d A(u)}{\left|\omega_{\Lambda, \Theta}(u)\right|^{2}}+\frac{1}{4 \pi \sin \pi \theta} \mathbf{Q}_{\Theta}\left(J_{1}, \ldots, J_{n-1}\right),
$$

where the quadratic form $\mathbf{Q}_{\Theta}$ is defined by (3.18).

Remark. If $a$ is an arbitrary point in $\Omega$, then we can choose formally $\lambda_{n+1}=a, \theta_{n+1}=0$ and then apply Theorem 3.1 to $\Lambda^{\prime}=\left\{\lambda_{1}, \ldots, \lambda_{n}, a\right\}$ and $\Theta^{\prime}=\left\{\theta_{1}, \ldots, \theta_{n}, 0\right\}$. Then we obtain

$$
[H, H]=\int_{\mathbb{D}}\left|x(u)^{2}\right| \frac{d A(u)}{\left|\omega_{\Lambda, \Theta}(u)\right|^{2}}+\frac{1}{4 \pi \sin \pi \theta} \mathbf{Q}_{\Theta^{\prime}}\left(J_{1}^{\prime}, \ldots, J_{n}^{\prime}\right),
$$


where

$$
J_{k}^{\prime}=\left(1-e^{-2 \pi i \theta_{k}}\right) e^{-2 \pi i\left(\theta_{1}+\cdots+\theta_{k-1}\right)} \int_{a}^{\lambda_{k}} h_{0}(u) d u, \quad k=1, \ldots, n .
$$

We turn to the case where $\theta=\theta_{1}+\cdots+\theta_{n}$ is an integer. We keep the same notation as before (except for $C$, which can be an arbitrary constant). Now the function $H$ is not uniquely determined by $h$. Choosing an arbitrary constant $C$ and then taking $H$ to be a primitive function to $h$ satisfying $H\left(\lambda_{n}^{*}\right)=C$, we obtain a function that is $(\Gamma, 0)$ character-automorphic for any simple path $\Gamma$ enclosing the points $\left\{\lambda_{1}, \ldots, \lambda_{n}\right\}$, because

$$
\int_{\Gamma} h(u) d u=0
$$

(see the discussion after Lemma 3.2). This identity can be rewritten as

$$
\sum_{k=1}^{n-1}\left(1-e^{-2 \pi i \theta_{k}}\right) e^{-2 \pi i\left(\theta_{1}+\cdots+\theta_{k-1}\right)} I_{k}=0 .
$$

Formulas (3.10)-(3.16) remain valid without changes, and we obtain

$$
\begin{aligned}
\int_{\gamma} h(u) \overline{H(u)} \frac{d u}{2 \pi i} \\
=\frac{1}{2 \pi i}\left(\sum_{k=1}^{n-1}\left(1-e^{-2 \pi i \theta_{k}}\right) e^{-2 \pi i\left(\theta_{1}+\cdots+\theta_{k-1}\right)} I_{k}\right) \cdot \bar{C} \\
\quad+\frac{1}{2 \pi i} \sum_{k=1}^{n-1}\left(1-e^{-2 \pi i \theta_{k}}\right) e^{-2 \pi i\left(\theta_{1}+\cdots+\theta_{k-1}\right)} I_{k} \cdot \sum_{l=1}^{k-1}\left(1-e^{2 \pi i \theta_{l}}\right) e^{2 \pi i\left(\theta_{1}+\cdots+\theta_{l-1}\right)} \overline{I_{l}} \\
\quad+\sum_{k=1}^{n-1} \frac{1-e^{-2 \pi i \theta_{k}}}{2 \pi i}\left|I_{k}\right|^{2} .
\end{aligned}
$$

As before, we define $J_{k}$ by (3.17), and we observe that (3.22) reduces to

$$
J_{1}+\cdots+J_{n-1}=0 .
$$

Then expression (3.23) becomes

$$
\sum_{k=1}^{n-1} \frac{\left|J_{k}\right|^{2}}{2 \pi i\left(1-e^{2 \pi i \theta_{k}}\right)}+\frac{1}{2 \pi i} \sum_{1 \leq l<k \leq n-1} J_{k} \overline{J_{l}} .
$$

Subtracting the relation

$$
0=\frac{1}{4 \pi i}\left|J_{1}+\cdots+J_{n-1}\right|^{2}=\frac{1}{4 \pi i} \sum_{1 \leq l, k \leq n-1} J_{k} \overline{J_{l}}
$$

from this formula, we finally get

$$
\int_{\gamma} h(u) \overline{H(u)} \frac{d u}{2 \pi i}=\frac{1}{4 \pi} \tilde{\mathbf{Q}}_{\Theta}\left(J_{1}, \ldots, J_{n-1}\right),
$$

where

$$
\widetilde{\mathbf{Q}}_{\Theta}\left(J_{1}, \ldots, J_{n-1}\right):=\sum_{k=1}^{n-1} \cot \pi \theta_{k}\left|J_{k}\right|^{2}-2 \operatorname{Re}\left(i \sum_{1 \leq l<k \leq n-1} J_{k} \overline{J_{l}}\right) .
$$

Thus, we have proved the following statement. 
Theorem 3.2. Assume that $\theta=\theta_{1}+\cdots+\theta_{n}$ is an integer. Then $H \in \mathcal{G}_{\Lambda, \Theta}^{-}$if and only if there exists a function $x \in \mathcal{B}_{0}^{+}$such that

$$
\partial_{u} H(u)=h(u)=\frac{x(u)}{\omega_{\Lambda, \Theta}(u)}, \quad u \in \mathbb{D},
$$

and the integral of $h$ along any simple path enclosing the points $\left\{\lambda_{1}, \ldots, \lambda_{n}\right\}$ vanishes.

Moreover, let $\Omega, h_{0}$, and $J_{k}$ be as in Theorem 3.1. Then $J_{1}+\cdots+J_{n-1}=0$ and

$$
[H, H]=\int_{\mathbb{D}}|x(u)|^{2} \frac{d A(u)}{\left|\omega_{\Lambda, \Theta}(u)\right|^{2}}+\widetilde{\mathbf{Q}}_{\Theta}\left(J_{1}, \ldots, J_{n-1}\right),
$$

where the quadratic form $\widetilde{\mathbf{Q}}_{\Theta}$ is defined by (3.25).

The quadratic form $\mathbf{Q}_{\Theta}$ is positive definite whenever $\theta_{1}+\cdots+\theta_{n}=\theta \leq 1$. This follows from the next lemma by the Sylvester criterion.

Lemma 3.4. Let $\theta_{1}, \ldots, \theta_{m}$ and $\theta$ be real numbers such that $\theta_{k}, k=1, \ldots, m$, are not integers. Let $A=A\left(\theta_{1}, \ldots, \theta_{m} ; \theta\right)=\left(A_{k j}\right)_{1 \leq k, j \leq m}$ denote the matrix with entries

$$
A_{k j}= \begin{cases}\frac{\sin \pi\left(\theta-\theta_{k}\right)}{\sin \pi \theta_{k}} & \text { if } k=j ; \\ -e^{i \pi \theta} & \text { if } k<j ; \\ -e^{-i \pi \theta} & \text { if } k>j .\end{cases}
$$

Then

$$
\operatorname{det}\left[A\left(\theta_{1}, \ldots, \theta_{m} ; \theta\right)\right]=\frac{(\sin \pi \theta)^{m-1} \cdot \sin \pi\left(\theta-\theta_{1}-\cdots-\theta_{m}\right)}{\sin \pi \theta_{1} \cdot \cdots \cdot \sin \pi \theta_{m}} .
$$

Proof. Without loss of generality we may assume that all numbers $\theta-\left(\theta_{1}+\cdots+\theta_{k}\right)$, $k=1, \ldots, m-1$ are not integers. Replacing each row $A_{k}, k=2, \ldots, n$, of the matrix $A$ by the row

we obtain the matrix

$$
A_{k}+\frac{\sin \pi \theta_{1}}{\sin \pi\left(\theta-\theta_{1}\right)} e^{-i \pi \theta} A_{1},
$$

$$
\left(\begin{array}{cccc}
\frac{\sin \pi\left(\theta-\theta_{1}\right)}{\sin \pi \theta_{1}} & -e^{i \pi \theta} & \ldots & -e^{i \pi \theta} \\
0 & B_{11} & \ldots & B_{1, m-1} \\
\vdots & \vdots & \ddots & \vdots \\
0 & B_{m-1,1} & \ldots & B_{m-1, m-1}
\end{array}\right)
$$

where the new matrix $B$ is given by

$$
B=\frac{\sin \pi \theta}{\sin \pi\left(\theta-\theta_{1}\right)} A\left(\theta_{2}, \ldots, \theta_{m} ; \theta-\theta_{1}\right) .
$$

Hence,

$$
\operatorname{det}\left[A\left(\theta_{1}, \ldots, \theta_{m} ; \theta\right)\right]=\frac{\sin \pi \theta}{\sin \pi \theta_{1}} \operatorname{det}\left[A\left(\theta_{2}, \ldots, \theta_{m} ; \theta-\theta_{1}\right)\right] .
$$

Iterating this, we arrive at (3.27).

Corollary 3.1. If $\theta_{1}+\cdots+\theta_{n}=\theta \leq 1$, then the quadratic form $\mathbf{Q}_{\Theta}$ given by (3.18) is positive definite.

Proposition 3.1. If the $\theta_{k}, k=1, \ldots, n$, are positive and $\theta_{1}+\cdots+\theta_{n}=\theta=1$, then the quadratic form $\widetilde{\mathbf{Q}}_{\Theta}\left(J_{1}, \ldots, J_{n-1}\right)$ given by (3.25) is positive definite on the hyperplane

$$
J_{1}+\cdots+J_{n-1}=0 .
$$


Proof. Let $r \Theta$ be the sequence $\left\{r \theta_{1}, \ldots, r \theta_{n}\right\}$, where $r \in(0,1]$. By the preceding discussion, the quadratic form $\mathbf{Q}_{r \Theta}$ is positive definite for any $r \in(0,1]$. Moreover, $\mathbf{Q}_{\Theta}\left(J_{1}, \ldots, J_{n-1}\right)=0$ on the hyperplane (3.28). Therefore,

$$
-\left.\frac{\partial}{\partial r}\right|_{r=1}\left[\mathbf{Q}_{r \Theta}\left(J_{1}, \ldots, J_{n-1}\right)\right] \geq 0
$$

on the hyperplane (3.28). But the last expression is equal to $\pi \widetilde{\mathbf{Q}}\left(J_{1}, \ldots, J_{n-1}\right)$, which gives the desired result.

We have proved the following.

Theorem 3.3. If $\theta_{1}+\cdots+\theta_{n}=\theta \leq 1$, then

$$
[H, H] \geq 0
$$

for any $H \in \mathcal{G}_{\Lambda, \Theta}^{-}$.

Corollary 3.2. (a) If $\theta_{1}+\cdots+\theta_{n}=\theta<1$, then $\mathcal{G}_{\Lambda, \Theta}^{+}$is a maximal positive subspace (with respect to the Dirichlet form) in $\mathcal{G}_{\theta}$.

(b) If $\theta_{1}+\cdots+\theta_{n}=\theta=1$, then the subspace $\{C\}+\mathcal{G}_{\Lambda, \Theta}^{+}$is a maximal positive subspace in $\mathcal{G}_{1}\left(=\mathcal{G}_{0}\right)$. Here $\{C\}$ is the subspace of constant functions.

Proof. (a) If $\theta$ is not an integer, then $\mathcal{G}_{\theta}$ is a Krein space and, clearly, $\mathcal{G}_{\Lambda, \Theta}^{+}$is a closed positive subspace there. Since its orthogonal companion $\left(\mathcal{G}_{\Lambda, \Theta}^{+}\right)^{\perp}=\left(\mathcal{G}_{\Lambda, \Theta}^{-}\right)^{\sharp}$ is a negative subspace, Lemma V.4.5 of [3] implies the claim.

(b) If $\theta=1$, the subspace $\{C\}$ of constant functions is an isotropic subspace in $\mathcal{G}_{0}$ and the factorable $\mathcal{G}_{0} /\{C\}$ is a Krĕ̌n space. As before, $\{C\}+\mathcal{G}_{\Lambda, \Theta}^{+}$is a maximal positive subspace in $\mathcal{G}_{0} /\{C\}$; hence, it is also a maximal positive subspace in $\mathcal{G}_{0}$.

The results on the maximal uniformly positive subspaces of Krĕn spaces presented in [3. Chapter V] imply now that the subspaces $\mathcal{G}_{\Lambda, \Theta}^{+}$and $\left(\mathcal{G}_{\Lambda, \Theta}^{+}\right)^{\perp}=\left(\mathcal{G}_{\Lambda, \Theta}^{-}\right)^{\sharp}$ form a fundamental decomposition of $\mathcal{G}_{\theta}$ into a sum of orthogonal positive and negative subspaces in the case where $\theta_{1}+\cdots+\theta_{n}=\theta \leq 1$ (if $\theta=1$, we obtain first a decomposition of $\mathcal{G}_{0} /\{C\}$, and then we realize that the constant subspace $\{C\}$ is included in $\left.\left(\mathcal{G}_{\Lambda, \Theta}^{-}\right)^{\sharp}\right)$. Therefore, we have proved the following theorem.

Theorem 3.4. Let $\theta_{1}+\cdots+\theta_{n}=\theta \leq 1$. Then $\mathcal{G}_{\theta}$ is the direct orthogonal sum

$$
\mathcal{G}_{\theta}=\mathcal{G}_{\Lambda, \Theta}^{+} \dot{+}\left(\mathcal{G}_{\Lambda, \Theta}^{-}\right)^{\sharp}
$$

of the positive subspace $\mathcal{G}_{\Lambda, \Theta}^{+}$and the negative subspace $\left(\mathcal{G}_{\Lambda, \Theta}^{-}\right)^{\sharp}$.

Theorem 3.3 shows that if $\theta_{1}+\cdots+\theta_{n} \leq 1$, then the right-hand side of (3.20) or of (3.26) is a certain Hilbert space norm of a function $x \in \mathcal{B}_{0}^{+}$. This norm will be denoted by $\|\cdot\|_{\mathcal{B}_{\Lambda, \Theta}}$, and the corresponding scalar product by $\langle\cdot, \cdot\rangle_{\Lambda, \Theta}$. In the case where $\theta_{1}+\cdots+\theta_{n}<1$, the space $\mathcal{B}_{0}^{+}$supplied with the norm $\|\cdot\|_{\Lambda, \Theta}$ will be denoted by $\mathcal{B}_{\Lambda, \Theta}$. In the case where $\theta_{1}+\cdots+\theta_{n}=1$, the notation $\mathcal{B}_{\Lambda, \Theta}$ stands for the space of functions $x$ such that

$$
\partial_{u} H(u)=\frac{x(u)}{\omega_{\Lambda, \Theta}(u)}
$$

for some $H \in \mathcal{G}_{\Lambda, \Theta}^{-}$; this space is supplied with the norm $\|\cdot\|_{\mathcal{B}_{\Lambda, \Theta}}$. Let $k_{\Lambda, \Theta}^{-}$denote the reproducing kernel for the space $\mathcal{B}_{\Lambda, \Theta}$. In other words, $k_{\Lambda, \Theta}^{-}(\cdot, \mu)$ is a function analytic in $\mathbb{D}$ such that there exists a function $H_{\mu} \in \mathcal{G}_{\Lambda, \Theta}^{-}$with

$$
\partial_{u} H_{\mu}(u)=\frac{k_{\Lambda, \Theta}^{-}(u, \mu)}{\omega_{\Lambda, \Theta}(u)},
$$


and for any function $x$ satisfying (3.30) we have

$$
x(\mu)=\left[H, H_{\mu}\right]=\left\langle x, k_{\Lambda, \Theta}^{-}(\cdot, \mu)\right\rangle_{\Lambda, \Theta} .
$$

The kernel $k_{\Lambda, \Theta}^{-}$should be thought of as a Bergman-type kernel, because the norm $\|\cdot\|_{\mathcal{B}_{\Lambda, \Theta}}$ is a Bergman-type norm, while the kernel $k_{\Lambda, \Theta}^{+}$introduced earlier is a Dirichlettype kernel because $\|\cdot\|_{\Lambda, \Theta}$ is a Dirichlet-type norm. Later on in this section, we shall find a relationship between the kernels $k_{\Lambda, \Theta}^{-}$and $k_{\Lambda, \Theta}^{+}$. Both these kernel functions will appear in $\$ 5$ in connection with area theorems.

Our next goal is to find an explicit form of the decompositions (3.29) for some functions in $\mathcal{G}_{\theta}$.

Definition 3.3. For each $a \in \mathbb{D}$, we define

$$
f_{a}(\zeta)=f_{\Lambda, \Theta, a}(\zeta):=\frac{1}{\zeta-a}-\sum_{\nu=1}^{n} \frac{\theta_{\nu} \bar{\lambda}_{\nu}}{1-a \bar{\lambda}_{\nu}} k_{\Lambda, \Theta}^{+}\left(\zeta, \lambda_{\nu}\right)
$$

as an element of $\mathcal{G}_{0}$.

Remark. In what follows in this section, we assume that $\Lambda$ and $\Theta$ are fixed and do not indicate the dependence of $f_{a}$ on $\Lambda$ and $\Theta$ explicitly.

Lemma 3.5. For each $a \in \mathbb{D}$, the function $\omega_{\Lambda, \Theta} f_{a}$ is orthogonal to $\mathcal{G}_{\Lambda, \Theta}^{+}$.

Proof. Let $g$ be an arbitrary function in $\mathcal{G}_{0}^{+}$. Then

$$
\begin{aligned}
& {\left[\omega_{\Lambda, \Theta} f_{a}, \omega_{\Lambda, \Theta} g\right]} \\
& \quad=\left[\frac{\omega_{\Lambda, \Theta}(\zeta)}{\zeta-a}, \omega_{\Lambda, \Theta}(\zeta) g(\zeta)\right]-\sum_{\nu=1}^{n} \frac{\theta_{\nu} \bar{\lambda}_{\nu}}{1-a \bar{\lambda}_{\nu}}\left[\omega_{\Lambda, \Theta} k_{\Lambda, \Theta}^{+}\left(\cdot, \lambda_{\nu}\right), \omega_{\Lambda, \Theta} g\right] \\
& \quad=\int_{\mathbb{T}}\left(\sum_{\nu=1}^{n} \theta_{\nu} \frac{1-\left|\lambda_{\nu}\right|^{2}}{\left|1-\bar{\lambda}_{\nu} z\right|^{2}}\right) \frac{\overline{g(z)}}{z-a} d m(z)+\left[\frac{1}{\zeta-a}, g(\zeta)\right]-\sum_{\nu=1}^{n} \frac{\theta_{\nu} \bar{\lambda}_{\nu} \overline{1-a \bar{\lambda}_{\nu}} \overline{g\left(\lambda_{\nu}\right)}}{\quad=\int_{\mathbb{T}}\left(\sum_{\nu=1}^{n} \theta_{\nu} \frac{1-\left|\lambda_{\nu}\right|^{2}}{\left|1-\bar{\lambda}_{\nu} z\right|^{2}}\right) \overline{\left(\frac{z g(z)}{1-\bar{a} z}\right)} d m(z)-\sum_{\nu=1}^{n} \frac{\theta_{\nu} \bar{\lambda}_{\nu}}{1-a \bar{\lambda}_{\nu}} \overline{g\left(\lambda_{\nu}\right)}=0 .}
\end{aligned}
$$

Corollary 3.3. The function $\frac{\omega_{\Lambda, \Theta}(\zeta)}{\zeta-a}$ decomposes with respect to (3.29) as

$$
\frac{\omega_{\Lambda, \Theta}(\zeta)}{\zeta-a}=\omega_{\Lambda, \Theta}(\zeta)\left[\sum_{\nu=1}^{n} \frac{\theta_{\nu} \bar{\lambda}_{\nu}}{1-a \bar{\lambda}_{\nu}} k_{\Lambda, \Theta}^{+}\left(\zeta, \lambda_{\nu}\right)\right]+\omega_{\Lambda, \Theta}(\zeta) f_{a}(\zeta) .
$$

Lemma 3.6. Let $H \in \mathcal{G}_{\Lambda, \Theta}^{-}$, and let $x \in \mathcal{B}_{\Lambda, \Theta}$ be defined by (3.30). Then

$$
-\left[H^{\sharp}, \omega_{\Lambda, \Theta} f_{a}\right]=\overline{x(a)}
$$

for any $a \in \mathbb{D}$.

Proof. We have

$$
\begin{aligned}
- & {\left[H^{\sharp}, \omega_{\Lambda, \Theta} f_{a}\right]=-\int_{\mathbb{T}} z \partial_{z} H^{\sharp}(z) \cdot \overline{\omega_{\Lambda, \Theta}(z) f_{a}(z)} d m(z) } \\
& =\int_{\mathbb{T}} \bar{z} \omega_{\Lambda, \Theta}(z) \overline{x(z)} \overline{\omega_{\Lambda, \Theta}(z)}\left(\frac{1}{\bar{z}-\bar{a}}-\sum_{\nu=1}^{n} \frac{\bar{\theta}_{\nu} \lambda_{\nu}}{1-\bar{a} \lambda_{\nu}} \overline{k_{\Lambda, \Theta}^{+}\left(z, \lambda_{\nu}\right)}\right) d m(z) \\
& =\int_{\mathbb{T}} \frac{\bar{z} \overline{x(z)}}{\bar{z}-\bar{a}} d m(z)=\overline{x(a)} .
\end{aligned}
$$

As a corollary, we obtain the following fact. 
Proposition 3.2. If $\theta_{1}+\cdots+\theta_{n} \leq 1$, then

$$
\partial_{u}\left(\omega_{\Lambda, \Theta} f_{a}\right)^{\sharp}(u)=\frac{k_{\Lambda, \Theta}^{-}(u, a)}{\omega_{\Lambda, \Theta}(u)}
$$

for any $a \in \mathbb{D}$.

Proof. Since $\left(\omega_{\Lambda, \Theta} f_{a}\right)^{\sharp} \in \mathcal{G}_{\Lambda, \Theta}^{-}$, we have

$$
\partial_{u}\left(\omega_{\Lambda, \Theta} f_{a}\right)^{\sharp}(u)=\frac{x_{a}(u)}{\omega_{\Lambda, \Theta}(u)}
$$

for some function $x_{a} \in \mathcal{B}_{\Lambda, \Theta}$. But then for any function $x \in \mathcal{B}_{\Lambda, \Theta}$ satisfying (3.30) we obtain

$$
\left\langle x, x_{a}\right\rangle_{\Lambda, \Theta}=\left[H,\left(\omega_{\Lambda, \Theta} f_{a}\right)^{\sharp}\right]=-\left[\omega_{\Lambda, \Theta} f_{a}, H^{\sharp}\right]=x(a),
$$

which shows that $x_{a}=k_{\Lambda, \Theta}^{-}(\cdot, a)$.

Finally, we get another corollary.

Corollary 3.4. If $\theta_{1}+\cdots+\theta_{n} \leq 1$, then

$$
-\left[\omega_{\Lambda, \Theta} f_{a}, \omega_{\Lambda, \Theta} f_{b}\right]=k_{\Lambda, \Theta}^{-}(a, b)
$$

for any $a, b \in \mathbb{D}$.

Proof. We have

$$
\begin{aligned}
-\left[\omega_{\Lambda, \Theta} f_{a}, \omega_{\Lambda, \Theta} f_{b}\right] & =\left[\left(\omega_{\Lambda, \Theta} f_{b}\right)^{\sharp},\left(\omega_{\Lambda, \Theta} f_{a}\right)^{\sharp}\right] \\
& =\left\langle k_{\Lambda, \Theta}^{-}(\cdot, b), k_{\Lambda, \Theta}^{-}(\cdot, a)\right\rangle_{\Lambda, \Theta}=k_{\Lambda, \Theta}^{-}(a, b) .
\end{aligned}
$$

The above inner product can be calculated explicitly in terms of the kernel $k_{\Lambda, \Theta}^{+}$. We formulate this as a separate statement.

Proposition 3.3. We have

$$
\begin{aligned}
k_{\Lambda, \Theta}^{-}(a, b)= & -\left[\omega_{\Lambda, \Theta} f_{a}, \omega_{\Lambda, \Theta} f_{b}\right] \\
= & \frac{1}{(1-\bar{b} a)^{2}}-\sum_{\nu=1}^{n} \theta_{k} \frac{1-\left|\lambda_{\nu}\right|^{2} \bar{b} a}{\left(1-\bar{b} \lambda_{\nu}\right)\left(1-a \bar{\lambda}_{\nu}\right)(1-\bar{b} a)} \\
& +\sum_{1 \leq \nu, l \leq n} \frac{\theta_{\nu} \theta_{l} \bar{\lambda}_{\nu} \lambda_{l}}{\left(1-a \bar{\lambda}_{\nu}\right)\left(1-\bar{b} \lambda_{l}\right)} k_{\Lambda, \Theta}^{+}\left(\lambda_{l}, \lambda_{\nu}\right) .
\end{aligned}
$$

Proof. Since all functions $k_{\Lambda, \Theta}^{+}\left(\cdot, \lambda_{\nu}\right)$ are orthogonal to $f_{b}$, we can write

$$
\begin{aligned}
& -\left[\omega_{\Lambda, \Theta} f_{a}, \omega_{\Lambda, \Theta} f_{b}\right]=-\left[\frac{\omega_{\Lambda, \Theta}(\zeta)}{\zeta-a}, \omega_{\Lambda, \Theta} f_{b}\right] \\
& =-\int_{\mathbb{T}} z \partial_{z}\left(\frac{\omega_{\Lambda, \Theta}(z)}{z-a}\right) \cdot \overline{\omega_{\Lambda, \Theta}(z) f_{b}(z)} d m(z) \\
& =-\int_{\mathbb{T}}\left(-\frac{z}{(z-a)^{2}}+\sum_{\nu=1}^{n} \theta_{\nu} \frac{z\left(1-\left|\lambda_{\nu}\right|^{2}\right)}{\left(z-\lambda_{\nu}\right)\left(1-\bar{\lambda}_{\nu} z\right)(z-a)}\right) \overline{f_{b}(z)} d m(z) .
\end{aligned}
$$

Using the partial fraction expansion

$$
\frac{z\left(1-\left|\lambda_{\nu}\right|^{2}\right)}{\left(z-\lambda_{\nu}\right)\left(1-\bar{\lambda}_{\nu} z\right)(z-a)}=\frac{\frac{\lambda_{\nu}}{\lambda_{\nu}-a}}{z-\lambda_{\nu}}+\frac{\frac{a\left(1-\left|\lambda_{\nu}\right|^{2}\right)}{\left(a-\lambda_{\nu}\right)\left(1-\bar{\lambda}_{\nu} a\right)}}{z-a}+\frac{\frac{\bar{\lambda}_{\nu}}{1-\lambda_{\nu} a}}{1-\bar{\lambda}_{\nu} z}
$$


and calculating the integrals

$$
\begin{aligned}
\int_{\mathbb{T}} \frac{\overline{f_{b}(z)}}{z-\mu} d m(z)=\frac{1}{1-\bar{b} \mu}, \\
\int_{\mathbb{T}} \frac{z \overline{f_{b}(z)}}{(z-a)^{2}} d m(z)=\frac{1}{(1-\bar{b} a)^{2}}, \\
\int_{\mathbb{T}} \frac{\overline{f_{b}(z)}}{1-\bar{\lambda} z} d m(z)=-\sum_{l=1}^{n} \frac{\theta_{l} \lambda_{l}}{1-\bar{b} \lambda_{l}} k_{\Lambda, \Theta}^{+}\left(\lambda_{l}, \lambda\right),
\end{aligned}
$$

we arrive at (3.36).

\section{§4. Domination of Kernels}

In this section we develop an abstract language of domination relations for kernel functions. It will allow us to formulate area theorems of a different type in a concise form avoiding too long inequalities.

Let $\mathcal{X}, \mathcal{Y}$ be some abstract sets, and let the functions $L(x, y), K_{1}\left(x, x^{\prime}\right)$, and $K_{2}\left(y, y^{\prime}\right)$ be such that

- $K_{1}\left(x, x^{\prime}\right)$ is defined on $\mathcal{X} \times \mathcal{X}$ and positive definite;

- $K_{2}\left(y, y^{\prime}\right)$ is defined on $\mathcal{Y} \times \mathcal{Y}$ and positive definite;

- $L(x, y)$ is defined on $\mathcal{X} \times \mathcal{Y}$.

Definition 4.1. In the above context, we shall say that the kernel $L(x, y)$ is dominated by the pair $\left\{K_{1}, K_{2}\right\}$ and write

$$
L(x, y) \ll\left\{K_{1}\left(x, x^{\prime}\right) ; K_{2}\left(y, y^{\prime}\right)\right\}
$$

if for any choice of $x_{1}, \ldots, x_{m} \in \mathcal{X}, y_{1}, \ldots, y_{n} \in \mathcal{Y}, \alpha_{1}, \ldots, \alpha_{m}, \beta_{1}, \ldots, \beta_{n} \in \mathbb{C}$ we have the following inequality:

$$
\begin{aligned}
& \left|\sum_{j=1}^{n} \sum_{i=1}^{m} \alpha_{i} \bar{\beta}_{j} L\left(x_{i}, y_{j}\right)\right|^{2} \\
& \quad \leq\left(\sum_{i=1}^{m} \sum_{i^{\prime}=1}^{m} \alpha_{i} \bar{\alpha}_{i^{\prime}} K_{1}\left(x_{i}, x_{i^{\prime}}\right)\right)\left(\sum_{j=1}^{n} \sum_{j^{\prime}=1}^{n} \beta_{j} \bar{\beta}_{j^{\prime}} K_{2}\left(y_{j}, y_{j^{\prime}}\right)\right) .
\end{aligned}
$$

An example of a domination relation is given by the Lebedev inequalities (see [5, p. 125])

$$
\begin{aligned}
& \left|\sum_{i=1}^{N} \sum_{j=1}^{N} \lambda_{i} \mu_{j} \log \left(\frac{g\left(z_{i}\right)-g\left(\zeta_{j}\right)}{z_{i}-\zeta_{j}}\right)\right|^{2} \\
& \quad \leq\left\{-\sum_{i=1}^{N} \sum_{j=1}^{N} \lambda_{i} \bar{\lambda}_{j} \log \left(1-\frac{1}{z_{i} \bar{z}_{j}}\right)\right\} \cdot\left\{-\sum_{i=1}^{N} \sum_{j=1}^{N} \mu_{i} \bar{\mu}_{j} \log \left(1-\frac{1}{\zeta_{i} \bar{\zeta}_{j}}\right)\right\},
\end{aligned}
$$

which is valid for the univalent functions $g$ in the class $\Sigma$ (i.e., for the functions $g$ univalent in $\mathbb{C} \backslash \overline{\mathbb{D}}$ and obeying the normalization $g(z)=z+O(1)$ at infinity). This family of inequalities is in fact the domination $L \ll\left\{K_{1} ; K_{2}\right\}$, where

$$
\begin{aligned}
L(z, \zeta) & =\log \left(\frac{g(z)-g(\zeta)}{z-\zeta}\right), \\
K_{1}\left(z, z^{\prime}\right) & =-\log \left(1-\frac{1}{z \bar{z}^{\prime}}\right), \\
K_{2}\left(\zeta, \zeta^{\prime}\right) & =-\log \left(1-\frac{1}{\bar{\zeta} \zeta^{\prime}}\right),
\end{aligned}
$$


and all variables are in $\mathbb{C} \backslash \overline{\mathbb{D}}$. It is well known that, by the symmetry of $L$ and the relation $K_{1}\left(z, z^{\prime}\right)=K_{2}\left(z^{\prime}, z\right)$, inequalities (4.3) are equivalent to the formally weaker Goluzin inequalities

$$
\left|\sum_{1 \leq i, j \leq N} \lambda_{i} \lambda_{j} \log \left(\frac{g\left(z_{i}\right)-g\left(z_{j}\right)}{z_{i}-z_{j}}\right)\right| \leq \sum_{1 \leq i, j \leq N}-\lambda_{i} \bar{\lambda}_{j} \log \left(1-\frac{1}{z_{i} \bar{z}_{j}}\right)
$$

(see, e.g., the discussion in [10, 3.6]).

Another example of domination inequalities (4.2) appears in the paper [2] (see inequality (4.14a) therein). It looks like this:

$$
l(\zeta, \eta) \ll\left\{K\left(\zeta, \zeta^{\prime}\right) ; K\left(\eta^{\prime}, \eta\right)-\Gamma\left(\eta^{\prime}, \eta\right)\right\},
$$

where

$$
\begin{aligned}
l(\zeta, \eta) & =\frac{1}{\pi(\zeta-\eta)^{2}}+\frac{2}{\pi} \frac{\partial^{2} G_{\Omega}(\zeta, \eta)}{\partial \zeta \partial \eta}, \\
K\left(\eta, \eta^{\prime}\right) & =-\frac{2}{\pi} \frac{\partial^{2} G_{\Omega}\left(\eta, \eta^{\prime}\right)}{\partial \eta \partial \bar{\eta}^{\prime}}, \\
\Gamma\left(\eta, \eta^{\prime}\right) & =\frac{1}{\pi} \int_{\mathbb{C} \backslash \Omega} \frac{d A(z)}{(z-\eta)^{2} \overline{\left(z-\eta^{\prime}\right)^{2}}},
\end{aligned}
$$

$G_{\Omega}(\cdot, \cdot)$ is the Green function for the Laplacian in a bounded smooth domain $\Omega \subset \mathbb{C}$, and all variables are in $\Omega$.

A requirement formally weaker than but equivalent to (4.2) is that for any choice of $x_{i}, y_{j}, \alpha_{j}$, and $\beta_{j}$ we have the inequality

$$
\left|\sum_{i, j} \alpha_{i} \bar{\beta}_{j} L\left(x_{i}, y_{j}\right)\right| \leq \frac{1}{2}\left(\sum_{i, i^{\prime}} \alpha_{i} \bar{\alpha}_{i^{\prime}} K_{1}\left(x_{i}, x_{i^{\prime}}\right)+\sum_{j, j^{\prime}} \beta_{j} \bar{\beta}_{j^{\prime}} K_{2}\left(y_{j}, y_{j^{\prime}}\right)\right) .
$$

Indeed, replacing in (4.5) $\alpha_{i}$ by $t \alpha_{i}$ and $\beta_{j}$ by $\beta_{j} / t$ with $t>0$ and then minimizing the right-hand side as a function of $t$, we obtain (4.2) (the converse implication is trivial).

As a consequence, we obtain the following property: if $L \ll\left\{K_{1} ; K_{2}\right\}$ and $\tilde{L} \ll$ $\left\{\tilde{K}_{1} ; \tilde{K}_{2}\right\}$, then $L+\tilde{L} \ll\left\{K_{1}+\tilde{K}_{1} ; K_{2}+\tilde{K}_{2}\right\}$.

The following theorem gives several equivalent conditions for the domination relation for kernel functions. For a positive definite kernel $K\left(x, x^{\prime}\right)$ with $x, x^{\prime} \in \mathcal{X}$, the standard notation $\mathcal{H}(K)$ stands for the Hilbert space of functions on $\mathcal{X}$ whose reproducing kernel is $K$.

Theorem 4.1. Let the kernels $L(x, y), K_{1}\left(x, x^{\prime}\right)$, and $K_{2}\left(y, y^{\prime}\right)$ be as above. Then the following conditions are equivalent:

(1) $L(x, y) \ll\left\{K_{1}\left(x, x^{\prime}\right) ; K_{2}\left(y, y^{\prime}\right)\right\}$;

(2) there is a contractive operator $T: \mathcal{H}\left(K_{2}\right) \mapsto \mathcal{H}\left(K_{1}\right)$ such that

$$
L(x, y)=\left(T K_{2}(\cdot, y), K_{1}(\cdot, x)\right)_{\mathcal{H}\left(K_{1}\right)}
$$

for any $x \in \mathcal{X}, y \in Y$;

(3a) $L(\cdot, y) \in \mathcal{H}\left(K_{1}\right)$ for any $y \in \mathcal{Y}$ and, moreover,

$$
\left\|\sum_{j=1}^{n} \bar{\beta}_{j} L\left(\cdot, y_{j}\right)\right\|_{\mathcal{H}\left(K_{1}\right)}^{2} \leq \sum_{1 \leq j, j^{\prime} \leq n} \beta_{j} \bar{\beta}_{j^{\prime}} K_{2}\left(y_{j}, y_{j^{\prime}}\right)
$$

for any choice of $y_{1}, \ldots, y_{n} \in \mathcal{Y}, \beta_{1}, \ldots, \beta_{n} \in \mathbb{C}$; 
(3b) $\overline{L(x, \cdot)} \in \mathcal{H}\left(K_{2}\right)$ for any $x \in \mathcal{X}$ and, moreover,

$$
\left\|\sum_{i=1}^{m} \bar{\alpha}_{i} \overline{L\left(x_{i}, \cdot\right)}\right\|_{\mathcal{H}\left(K_{2}\right)}^{2} \leq \sum_{1 \leq i, i^{\prime} \leq m} \alpha_{i} \bar{\alpha}_{i^{\prime}} K_{1}\left(x_{i}, x_{i^{\prime}}\right)
$$

for any choice of $x_{1}, \ldots, x_{m} \in \mathcal{X}, \alpha_{1}, \ldots, \alpha_{m} \in \mathbb{C}$.

Proof. We start with the implication $(1) \Longrightarrow(2)$. First, we prove that $L(\cdot, y) \in \mathcal{H}\left(K_{1}\right)$ for any $y \in \mathcal{Y}$. Let $y$ be fixed. From (4.2) it follows that

$$
\begin{aligned}
\left|\sum_{i=1}^{n} \alpha_{i} L\left(x_{i}, y\right)\right| & \leq K_{2}^{1 / 2}(y, y)\left(\sum_{i, i^{\prime}} \alpha_{i} \bar{\alpha}_{i^{\prime}} K_{1}\left(x_{i}, x_{i^{\prime}}\right)\right)^{1 / 2} \\
& =K_{2}^{1 / 2}(y, y)\left\|\sum_{i^{\prime}} \bar{\alpha}_{i^{\prime}} K_{1}\left(\cdot, x_{i^{\prime}}\right)\right\|_{\mathcal{H}\left(K_{1}\right)} .
\end{aligned}
$$

Therefore, the linear mapping

$$
\lambda_{y}: \sum_{i} \bar{\alpha}_{i} K_{1}\left(\cdot, x_{i}\right) \mapsto \sum_{i} \bar{\alpha}_{i} \overline{L\left(x_{i}, y\right)}
$$

extends to a bounded linear functional on $\mathcal{H}\left(K_{1}\right)$; hence, it is determined by a scalar product with some function $l_{y} \in \mathcal{H}\left(K_{1}\right)$. Since

$$
\overline{l_{y}(x)}=\left(K_{1}(\cdot, x), l_{y}\right)_{\mathcal{H}\left(K_{1}\right)}=\lambda_{y}\left(K_{1}(\cdot, x)\right)=\overline{L(x, y)},
$$

we obtain $L(\cdot, y)=l_{y} \in \mathcal{H}\left(K_{1}\right)$.

Now, we define an operator $T: \mathcal{H}\left(K_{2}\right) \mapsto \mathcal{H}\left(K_{1}\right)$ first on finite linear combinations of the functions $K_{2}(\cdot, y)$ as follows:

$$
T\left(\sum_{j} \bar{\beta}_{j} K_{2}\left(\cdot, y_{j}\right)\right):=\sum_{j} \bar{\beta}_{j} L\left(\cdot, y_{j}\right) .
$$

Then

$$
\begin{aligned}
& \left|\left(T\left(\sum_{j} \bar{\beta}_{j} K_{2}\left(\cdot, y_{j}\right)\right), \sum_{i} \bar{\alpha}_{i} K_{1}\left(\cdot, x_{i}\right)\right)_{\mathcal{H}\left(K_{1}\right)}\right| \\
& \quad=\left|\sum_{i, j} \alpha_{i} \bar{\beta}_{j} L\left(x_{i}, y_{j}\right)\right| \leq\left(\sum_{i, i^{\prime}} \alpha_{i} \bar{\alpha}_{i^{\prime}} K_{1}\left(x_{i}, x_{i^{\prime}}\right)\right)^{1 / 2} \cdot\left(\sum_{j, j^{\prime}} \beta_{j} \bar{\beta}_{j^{\prime}} K_{2}\left(y_{j}, y_{j^{\prime}}\right)\right)^{1 / 2} \\
& \quad=\left\|\sum_{i} \bar{\alpha}_{i} K_{1}\left(\cdot, x_{i}\right)\right\| \cdot\left\|\sum_{j} \bar{\beta}_{j} K_{2}\left(\cdot, y_{j}\right)\right\|,
\end{aligned}
$$

which shows that $T$ can be extended to a contractive operator from $\mathcal{H}\left(K_{2}\right)$ to $\mathcal{H}\left(K_{1}\right)$.

To prove the implication $(2) \Longrightarrow(3 \mathrm{a})$, we observe that the assumption

$$
L(\cdot, y)=T K_{2}(\cdot, y),
$$

equivalent to (4.6), implies

$$
\left\|\sum_{j=1}^{n} \bar{\beta}_{j} L\left(\cdot, y_{j}\right)\right\|_{\mathcal{H}\left(K_{1}\right)}^{2} \leq\|T\|^{2}\left\|\sum_{j} \bar{\beta}_{j} K_{2}\left(\cdot, y_{j}\right)\right\|_{\mathcal{H}\left(K_{2}\right)}^{2}=\|T\|^{2} \sum_{j, j^{\prime}} \beta_{j} \bar{\beta}_{j^{\prime}} K_{2}\left(y_{j}, y_{j^{\prime}}\right) .
$$


Finally, (3a) implies (1) because

$$
\begin{gathered}
\left|\sum_{i, j} \alpha_{i} \bar{\beta}_{j} L\left(x_{i}, y_{j}\right)\right|^{2}=\left|\left(\sum_{j} \bar{\beta}_{j} L\left(\cdot, y_{j}\right), \sum_{i} \bar{\alpha}_{i} K_{1}\left(\cdot, x_{i}\right)\right)_{\mathcal{H}\left(K_{1}\right)}\right|^{2} \\
\leq\left\|\sum_{j} \bar{\beta}_{j} L\left(\cdot, y_{j}\right)\right\|_{\mathcal{H}\left(K_{1}\right)}^{2} \cdot\left\|\sum_{i} \bar{\alpha}_{i} K_{1}\left(\cdot, x_{i}\right)\right\|_{\mathcal{H}\left(K_{1}\right)}^{2} \\
\leq\left(\sum_{i=1}^{m} \sum_{i^{\prime}=1}^{m} \alpha_{i} \bar{\alpha}_{i^{\prime}} K_{1}\left(x_{i}, x_{i^{\prime}}\right)\right)\left(\sum_{j=1}^{n} \sum_{j^{\prime}=1}^{n} \beta_{j} \bar{\beta}_{j^{\prime}} K_{2}\left(y_{j}, y_{j^{\prime}}\right)\right) .
\end{gathered}
$$

The obvious modifications needed to prove the implications $(2) \Longrightarrow(3 \mathrm{~b}) \Longrightarrow$ (1) are left to the reader.

We saw above that domination relations can be added. The next proposition shows that they can be multiplied as well.

Proposition 4.1. Assume that we have the dominations

$$
L(x, y) \ll\left\{K_{1}\left(x, x^{\prime}\right) ; K_{2}\left(y, y^{\prime}\right)\right\} \quad \text { and } \quad \tilde{L}(x, y) \ll\left\{\tilde{K}_{1}\left(x, x^{\prime}\right) ; \tilde{K}_{2}\left(y, y^{\prime}\right)\right\},
$$

where $x, x^{\prime} \in \mathcal{X}, y, y^{\prime} \in \mathcal{Y}$.

Then

$$
L(x, y) \tilde{L}(x, y) \ll\left\{K_{1}\left(x, x^{\prime}\right) \tilde{K}_{1}\left(x, x^{\prime}\right) ; K_{2}\left(y, y^{\prime}\right) \tilde{K}_{2}\left(y, y^{\prime}\right)\right\} .
$$

Proof. The proof is completely similar to that of the classical Schur theorem on elementwise products of positive definite matrices.

By Theorem 4.1, there exist contractions $T: \mathcal{H}\left(K_{2}\right) \mapsto \mathcal{H}\left(K_{1}\right)$ and $\tilde{T}: \mathcal{H}\left(\tilde{K}_{2}\right) \mapsto$ $\mathcal{H}\left(\tilde{K}_{1}\right)$ such that

$$
L(x, y)=\left(T K_{2}(\cdot, y), K_{1}(\cdot, x)\right)_{\mathcal{H}\left(K_{1}\right)} \quad \text { and } \quad \tilde{L}(\tilde{x}, \tilde{y})=\left(\tilde{T} \tilde{K}_{2}(\cdot, \tilde{y}), \tilde{K}_{1}(\cdot, \tilde{x})\right)_{\mathcal{H}\left(\tilde{K}_{1}\right)} .
$$

Let $T \otimes \tilde{T}: \mathcal{H}\left(K_{2}\right) \otimes \mathcal{H}\left(\tilde{K}_{2}\right) \mapsto \mathcal{H}\left(K_{1}\right) \otimes \mathcal{H}\left(\tilde{K}_{1}\right)$ denote the tensor product of $T$ and $\tilde{T}$. Then

$$
L(x, y) \tilde{L}(\tilde{x}, \tilde{y})=\left(T \otimes \tilde{T}\left(K_{2}(\cdot, y) \otimes \tilde{K}_{2}(\cdot, \tilde{y})\right), K_{1}(\cdot, x) \otimes \tilde{K}_{1}(\cdot, \tilde{x})\right)_{\mathcal{H}\left(K_{1}\right) \otimes \mathcal{H}\left(\tilde{K}_{1}\right)},
$$

which implies the domination

$$
L(x, y) \tilde{L}(\tilde{x}, \tilde{y}) \ll\left\{K_{1}\left(x, x^{\prime}\right) \tilde{K}_{1}\left(\tilde{x}, \tilde{x}^{\prime}\right) ; K_{2}\left(y, y^{\prime}\right) \tilde{K}_{2}\left(\tilde{y}, \tilde{y}^{\prime}\right)\right\} .
$$

Restricting it to the diagonal $x=\tilde{x}, y=\tilde{y}, x^{\prime}=\tilde{x}^{\prime}, y^{\prime}=\tilde{y}^{\prime}$, we obtain the desired conclusion.

Combining addition and multiplication of dominations, we obtain, in particular, the following.

Corollary 4.1. The domination $L(x, y) \ll\left\{K_{1}\left(x, x^{\prime}\right) ; K_{2}\left(y, y^{\prime}\right)\right\}$ implies that

$$
\exp (c L(x, y)) \ll\left\{\exp \left(c K_{1}\left(x, x^{\prime}\right)\right) ; \exp \left(c K_{2}\left(y, y^{\prime}\right)\right)\right\}
$$

for any $c>0$.

In the case of the domination (4.3), the above corollary is a well-known result on exponentiation of Goluzin inequalities (see, e.g., [5, Chapter 5]).

The next proposition shows how to differentiate domination relations. 
Proposition 4.2. Assume that

$$
L(z, y) \ll\left\{K_{1}\left(z, z^{\prime}\right) ; K_{2}\left(y, y^{\prime}\right)\right\}, \quad z, z^{\prime} \in \Omega, y, y^{\prime} \in \mathcal{Y},
$$

where $\Omega$ is a domain in $\mathbb{C}$. Assume also that $K_{1}$ is differentiable with respect to the variables $z$ and $\bar{z}^{\prime}$. Then $L(z, y)$ is differentiable with respect to the variable $z$ for any $y \in \mathcal{Y}$, and

$$
\frac{\partial L}{\partial z}(z, y) \ll\left\{\frac{\partial^{2} K_{1}\left(z, z^{\prime}\right)}{\partial z \partial \bar{z}^{\prime}} ; K_{2}\left(y, y^{\prime}\right)\right\} .
$$

Remark. Of course, a similar result is true for differentiation with respect to the variable $y$.

Proof. The property that $K_{1}$ is differentiable with respect to $z$ and $\bar{z}^{\prime}$ implies that all functions in $\mathcal{H}\left(K_{1}\right)$ are differentiable in $\Omega$. Moreover, the mapping $\bar{z} \mapsto K_{1}(\cdot, z)$ is differentiable as an $\mathcal{H}\left(K_{1}\right)$-valued function defined on $\bar{\Omega}$, and for any $f \in \mathcal{H}\left(K_{1}\right)$ we have

$$
\partial_{z} f(z)=\left(f, \partial_{\bar{z}} K_{1}(\cdot, z)\right)_{\mathcal{H}\left(K_{1}\right)} .
$$

Since $L(\cdot, y) \in \mathcal{H}\left(K_{1}\right)$ by Theorem 4.1, we deduce that $L(z, y)$ is differentiable with respect to $z$ and, moreover,

$$
\partial_{z} L(z, y)=\left(L(\cdot, y), \partial_{\bar{z}} K_{1}(\cdot, z)\right)_{\mathcal{H}\left(K_{1}\right)} .
$$

By Theorem 4.1, there exists a contraction $T: \mathcal{H}\left(K_{2}\right) \mapsto \mathcal{H}\left(K_{1}\right)$ such that $L(\cdot, y)=$ $T K_{2}(\cdot, y)$. We obtain therefore

$$
\partial_{z} L(z, y)=\left(T K_{2}(\cdot, y), \partial_{\bar{z}} K_{1}(\cdot, z)\right)_{\mathcal{H}\left(K_{1}\right)} \cdot
$$

It remains to apply the same arguments as in the proof of the implication $(2) \Longrightarrow(3 \mathrm{a})$ of Theorem 4.1, together with the identity

$$
\left(\partial_{\bar{z}^{\prime}} K_{1}\left(\cdot, z^{\prime}\right), \partial_{\bar{z}} K_{1}(\cdot, z)\right)_{\mathcal{H}\left(K_{1}\right)}=\frac{\partial^{2} K_{1}\left(z, z^{\prime}\right)}{\partial z \partial \bar{z}^{\prime}} .
$$

Remark. The differentiation operator $\frac{\partial}{\partial z}$ can be replaced by more general differential (or integral) operators.

Definition 4.2. Assume that $L(x, y) \ll\left\{K_{1}\left(x, x^{\prime}\right) ; K_{2}\left(y, y^{\prime}\right)\right\}$. We shall say that this domination is exact if the operator $T: \mathcal{H}\left(K_{2}\right) \mapsto \mathcal{H}\left(K_{1}\right)$ such that $L(\cdot, y)=T K_{2}(\cdot, y)$ is a unitary operator.

The domination (4.3) given by the Lebedev inequalities is exact if and only if $g$ is a full mapping, i.e., it maps $\mathbb{C} \backslash \mathbb{D}$ onto a complement of an area-null set. Indeed, up to an appropriate normalization on Taylor coefficients, in this context the operator that maps $K_{2}(\cdot, \zeta)$ to $L(\cdot, \zeta)$ is the classical Grunsky operator, which is unitary if and only if $g$ is a full mapping.

The next proposition is almost obvious.

Proposition 4.3. The following conditions are equivalent:

(1) The domination $L(x, y) \ll\left\{K_{1}\left(x, x^{\prime}\right) ; K_{2}\left(y, y^{\prime}\right)\right\}$ is exact.

(2) The functions $L(\cdot, y), y \in \mathcal{Y}$, form a complete system of functions in $\mathcal{H}\left(K_{1}\right)$, and for any choice of $y_{1}, \ldots, y_{n} \in \mathcal{Y}$ and $\beta_{1}, \ldots, \beta_{n} \in \mathbb{C}$ we have

$$
\left\|\sum_{j=1}^{n} \bar{\beta}_{j} L\left(\cdot, y_{j}\right)\right\|_{\mathcal{H}\left(K_{1}\right)}^{2}=\sum_{1 \leq j, j^{\prime} \leq n} \beta_{j} \bar{\beta}_{j^{\prime}} K_{2}\left(y_{j}, y_{j^{\prime}}\right) .
$$


S. SHIMORIN

(3) The functions $\overline{L(x, \cdot)}, x \in \mathcal{X}$, form a complete system of functions in $\mathcal{H}\left(K_{2}\right)$, and for any choice of $x_{1}, \ldots, x_{m} \in \mathcal{X}$ and $\alpha_{1}, \ldots, \alpha_{m} \in \mathbb{C}$ we have

$$
\left\|\sum_{i=1}^{m} \bar{\alpha}_{i} \overline{L\left(x_{i}, \cdot\right)}\right\|_{\mathcal{H}\left(K_{2}\right)}^{2}=\sum_{1 \leq i, i^{\prime} \leq n} \alpha_{i} \bar{\alpha}_{i^{\prime}} K_{1}\left(x_{i}, x_{i^{\prime}}\right) .
$$

(4) The two identities (4.10) and (4.11) are true for any choice of parameters $x_{i}, y_{j}$, $\alpha_{i}$, and $\beta_{j}$.

Our last proposition of a technical nature can be proved by the same arguments as in the proof of Proposition 4.2. For $\theta>0$, the differential operator $D^{\theta}$ is defined as follows:

$$
D^{\theta}: g(z) \mapsto z^{1-\theta} \partial_{z}\left(z^{\theta} g(z)\right)=\theta g(z)+z \partial_{z} g(z) \text {. }
$$

Clearly, $D^{\theta}$ is invertible as an operator on functions analytic in $\mathbb{D}$, and the inverse operator is

$$
h(z) \mapsto \int_{0}^{1} t^{\theta-1} h(t z) d t .
$$

Proposition 4.4. Let $L(z, y), K_{1}\left(z, z^{\prime}\right)$, and $K_{2}\left(y, y^{\prime}\right)$ be as in Proposition 4.2. Then the domination

$$
L(z, y) \ll\left\{K_{1}\left(z, z^{\prime}\right) ; K_{2}\left(y, y^{\prime}\right)\right\}
$$

is exact if and only if the differentiated domination

$$
D_{z}^{\theta} L(z, y) \ll\left\{D_{z}^{\theta} D_{\bar{z}^{\prime}}^{\theta} K_{1}\left(z, z^{\prime}\right) ; K_{2}\left(y, y^{\prime}\right)\right\}
$$

is exact.

\section{§5. AREA THEOREMS}

In this section, we obtain two branching point area theorems (Theorems 5.1 and 5.2 below), which are the main results of this paper. Both theorems are formulated as certain domination relations for kernel functions. By using the equivalent conditions in Theorem 4.1. we can easily reformulate these theorems in terms of integral inequalities.

Let $\Lambda=\left\{\lambda_{1}, \ldots, \lambda_{n}\right\}$ be a fixed sequence of distinct points in $\mathbb{D}$, and let $\Theta=$ $\left\{\theta_{1}, \ldots, \theta_{n}\right\}$ be a fixed sequence of positive numbers such that $\theta=\theta_{1}+\cdots+\theta_{n} \leq 1$. We recall (see $\S 3$ ) that $k_{\Lambda, \Theta}^{+}(\cdot, \cdot)$ and $k_{\Lambda, \Theta}^{-}(\cdot, \cdot)$ are reproducing kernels relative to the norms defined by (3.2) and (3.20) (or (3.26) if $\theta_{1}+\cdots+\theta_{n}=1$ ), respectively. The functions $f_{\Lambda, \Theta, a}$ are defined by (3.31).

Theorem 5.1. Let $\psi: \mathbb{D} \mapsto \mathbb{D}$ be a conformal self-map of the unit disk. Then we have the domination

$$
l_{\psi, \Lambda, \Theta}(\zeta, u) \ll\left\{k_{\Lambda, \Theta}^{+}\left(\zeta, \zeta^{\prime}\right) ; \overline{k_{\Lambda, \Theta}^{-}\left(u, u^{\prime}\right)-k_{\psi, \Lambda, \Theta}^{-}\left(u, u^{\prime}\right)}\right\},
$$

where

$$
k_{\psi, \Lambda, \Theta}^{-}\left(u, u^{\prime}\right)=\frac{\omega_{\Lambda, \Theta}(u)}{\omega_{\psi(\Lambda), \Theta}(\psi(u))} \overline{\left(\frac{\omega_{\Lambda, \Theta}\left(u^{\prime}\right)}{\omega_{\psi(\Lambda), \Theta}\left(\psi\left(u^{\prime}\right)\right)}\right)} \psi^{\prime}(u) \overline{\psi^{\prime}\left(u^{\prime}\right)} k_{\psi(\Lambda), \Theta}^{-}\left(\psi(u), \psi\left(u^{\prime}\right)\right)
$$

and

$$
l_{\psi, \Lambda, \Theta}(\zeta, u)=\frac{\omega_{\psi(\Lambda), \Theta}(\psi(\zeta))}{\omega_{\Lambda, \Theta}(\zeta)} \frac{\omega_{\Lambda, \Theta}(u)}{\omega_{\psi(\Lambda), \Theta}(\psi(u))} \psi^{\prime}(u) f_{\psi(\Lambda), \Theta, \psi(u)}(\psi(\zeta))-f_{\Lambda, \Theta, u}(\zeta)
$$

(all variables are in $\mathbb{D})$. 
Proof. We pick $u_{1}, \ldots, u_{N} \in \mathbb{D}$ and $c_{1}, \ldots, c_{N} \in \mathbb{C}$ and consider the function

$$
g(\zeta)=\omega_{\psi(\Lambda), \Theta}(\zeta) \sum_{k=1}^{N} c_{k} \frac{\omega_{\Lambda, \Theta}\left(u_{k}\right)}{\omega_{\psi(\Lambda), \Theta}\left(\psi\left(u_{k}\right)\right)} \psi^{\prime}\left(u_{k}\right) f_{\psi(\Lambda), \Theta, \psi\left(u_{k}\right)}(\zeta) .
$$

By Corollary 3.4, we have

$$
-[g, g]=\sum_{k, l} c_{k} \bar{c}_{l} k_{\psi, \Lambda, \Theta}^{-}\left(u_{k}, u_{l}\right) .
$$

Proposition 2.2 implies that

$$
[g \circ \psi, g \circ \psi] \leq[g, g] .
$$

On the other hand, $g \circ \psi$ admits the following explicit decomposition into a sum of positive and negative terms related to (3.29):

$$
g \circ \psi=G_{+}+G_{-}
$$

where

$$
G_{+}(\cdot)=\omega_{\Lambda, \Theta}(\cdot) \sum_{k=1}^{N} c_{k} l_{\psi, \Lambda, \Theta}\left(\cdot, u_{k}\right)
$$

and

$$
G_{-}(\cdot)=\omega_{\Lambda, \Theta}(\cdot) \sum_{k=1}^{N} c_{k} f_{\Lambda, \Theta, u_{k}}(\cdot)
$$

so that

$$
[g \circ \psi, g \circ \psi]=\left[G_{+}, G_{+}\right]+\left[G_{-}, G_{-}\right]
$$

By Corollary 3.4 ,

$$
-\left[G_{-}, G_{-}\right]=\sum_{k, l} c_{k} \bar{c}_{l} k_{\Lambda, \Theta}^{-}\left(u_{k}, u_{l}\right) .
$$

Combining (5.4), (5.5), and (5.6), we obtain

$$
\begin{gathered}
{\left[\omega_{\Lambda, \Theta}(\cdot) \sum_{k=1}^{N} c_{k} l_{\psi, \Lambda, \Theta}\left(\cdot, u_{k}\right), \omega_{\Lambda, \Theta}(\cdot) \sum_{k=1}^{N} c_{k} l_{\psi, \Lambda, \Theta}\left(\cdot, u_{k}\right)\right]} \\
\leq \sum_{k, l} c_{k} \bar{c}_{l}\left(k_{\Lambda, \Theta}^{-}\left(u_{k}, u_{l}\right)-k_{\psi, \Lambda, \Theta}^{-}\left(u_{k}, u_{l}\right)\right) .
\end{gathered}
$$

By the definition of the kernel $k_{\Lambda, \Theta}^{+}$, this inequality is equivalent to the domination (5.1) by Theorem 4.1 .

Theorem 5.2. Let $\varphi$ be a function univalent in $\mathbb{D}$. Then we have the domination

$$
L_{\varphi, \Lambda, \Theta}(\zeta, u) \ll\left\{k_{\Lambda, \Theta}^{+}\left(\zeta, \zeta^{\prime}\right) ; \overline{k_{\Lambda, \Theta}^{-}\left(u, u^{\prime}\right)}\right\},
$$

where

(5.9)

$$
\begin{aligned}
& L_{\varphi, \Lambda, \Theta}(\zeta, u) \\
& \quad=\left[\prod_{k=1}^{n}\left(\frac{\varphi(\zeta)-\varphi\left(\lambda_{k}\right)}{\zeta-\lambda_{k}} \cdot \frac{1-\bar{\lambda}_{k} \zeta}{1-\bar{\lambda}_{k} u} \cdot \frac{u-\lambda_{k}}{\varphi(u)-\varphi\left(\lambda_{k}\right)}\right)^{\theta_{k}}\right] \frac{\varphi^{\prime}(u)}{\varphi(\zeta)-\varphi(u)}-f_{\Lambda, \Theta, u}(\zeta)
\end{aligned}
$$

and all variables are in $\mathbb{D}$. 
Proof. We argue as in the proof of Theorem 5.1. Suppose $u_{1}, \ldots, u_{N} \in \mathbb{D}$ and $c_{1}, \ldots, c_{N} \in$ $\mathbb{C}$. Consider the function

$$
\begin{aligned}
P(z)= & {\left[\prod_{k=1}^{n}\left(z-\varphi\left(\lambda_{k}\right)\right)^{\theta_{k}}\right] } \\
& \times \sum_{\nu=1}^{N}\left[\prod_{k=1}^{n}\left(\frac{u_{\nu}-\lambda_{k}}{\left(1-\bar{\lambda}_{k} u_{\nu}\right)\left(\varphi\left(u_{\nu}\right)-\varphi\left(\lambda_{k}\right)\right)}\right)^{\theta_{k}}\right] \cdot \frac{c_{\nu} \varphi^{\prime}\left(u_{\nu}\right)}{z-\varphi\left(u_{\nu}\right)} .
\end{aligned}
$$

By Corollary 2.2, we have

$$
[P \circ \varphi, P \circ \varphi] \leq 0
$$

with equality in the case where $\varphi$ is a full mapping. On the other hand, in accordance with (3.29), $P \circ \varphi$ can be written explicitly as $P \circ \varphi=G_{+}+G_{-}$, where

$$
G_{+}(\zeta)=\omega_{\Lambda, \Theta}(\zeta) \sum_{\nu=1}^{N} c_{\nu} L_{\varphi, \Lambda, \Theta}\left(\zeta, u_{\nu}\right)
$$

and

$$
G_{-}(\zeta)=\omega_{\Lambda, \Theta}(\zeta) \sum_{\nu=1}^{N} c_{\nu} f_{\Lambda, \Theta, u_{\nu}}(\zeta)
$$

so that

By Corollary 3.4 .

$$
[P \circ \varphi, P \circ \varphi]=\left[G_{+}, G_{+}\right]+\left[G_{-}, G_{-}\right] .
$$

$$
-\left[G_{-}, G_{-}\right]=\sum_{k, l} c_{k} \bar{c}_{l} k_{\Lambda, \Theta}^{-}\left(u_{k}, u_{l}\right)
$$

which implies

$$
\left[\omega_{\Lambda, \Theta}(\cdot) \sum_{\nu=1}^{N} c_{\nu} L_{\varphi, \Lambda, \Theta}\left(\cdot, u_{\nu}\right), \omega_{\Lambda, \Theta}(\cdot) \sum_{\nu=1}^{N} c_{\nu} L_{\varphi, \Lambda, \Theta}\left(\cdot, u_{\nu}\right)\right] \leq \sum_{\nu, l} c_{\nu} \bar{c}_{l} k_{\Lambda, \Theta}^{-}\left(u_{\nu}, u_{l}\right)
$$

(with equality if $\varphi$ is a full mapping). As in the proof of Theorem 5.1, this inequality is equivalent to the domination (5.8).

Our next aim is to prove that the domination (5.8) is exact if $\varphi$ is a full mapping. Without loss of generality, in the remaining part of this section we assume that $\varphi(0)=0$. First, we analyze the special case where the sequence $\Lambda$ reduces to a single point at the origin:

$$
\Lambda=\Lambda_{0}=\{0\} ; \quad \Theta_{0}=\{\theta\} .
$$

We consider the case of $\theta \in(0,1)$ only; slight modifications needed in the case where $\theta=1$ are left to the reader. Our analysis partially repeats that performed in [9], and we present it here for completeness.

By Lemma 3.1, the norm in the space $\mathcal{D}_{\Lambda_{0}, \Theta_{0}}$ is given by the formula

$$
\|g\|_{\mathcal{D}_{\Lambda_{0}, \Theta_{0}}}^{2}=\int_{\mathbb{D}}\left|\theta g(z)+z g^{\prime}(z)\right|^{2}|z|^{2 \theta-2} d A(z)=\sum_{k \geq 0}(k+\theta)|\hat{g}(k)|^{2} .
$$

By (3.20), the norm in $\mathcal{B}_{\Lambda_{0}, \Theta_{0}}$ is

$$
\|x\|_{\mathcal{B}_{\Lambda_{0}, \Theta_{0}}}^{2}=\int_{\mathbb{D}} \frac{|x(u)|^{2}}{|u|^{2 \theta}} d A(u)=\sum_{k \geq 0} \frac{|\hat{x}(k)|^{2}}{k+1-\theta} .
$$

In particular, we see that

$$
\|g\|_{\mathcal{D}_{\Lambda_{0}, \Theta_{0}}}^{2}=\left\|\theta g(z)+z g^{\prime}(z)\right\|_{\mathcal{B}_{\Lambda_{0}, 1-\Theta_{0}}}^{2}=\left\|D^{\theta} g\right\|_{\mathcal{B}_{\Lambda_{0}, 1-\Theta_{0}}}^{2},
$$


where $\mathbf{1}-\Theta_{0}=\{1-\theta\}$, and the differential operator $D^{\theta}$ is defined by (4.12). The explicit formulas (5.12) and (5.13) show that

$$
k_{\Lambda_{0}, \Theta_{0}}^{+}\left(\zeta, \zeta^{\prime}\right)=\sum_{k \geq 0} \frac{\left(\zeta \bar{\zeta}^{\prime}\right)^{k}}{k+\theta}
$$

and

$$
k_{\Lambda_{0}, \Theta_{0}}^{-}\left(u, u^{\prime}\right)=\sum_{k \geq 0}(k+1-\theta)\left(u \bar{u}^{\prime}\right)^{k}=D_{u}^{1-\theta} D_{\bar{u}^{\prime}}^{1-\theta} k_{\Lambda_{0}, \mathbf{1}-\Theta_{0}}^{+}\left(u, u^{\prime}\right) .
$$

Finally, the kernel $L_{\varphi, \Lambda_{0}, \Theta_{0}}$ (defined by (5.9) ) is of the form

$$
L_{\varphi, \Lambda_{0}, \Theta_{0}}(\zeta, u)=\left(\frac{\varphi(\zeta)}{\zeta} \frac{u}{\varphi(u)}\right)^{\theta} \frac{\varphi^{\prime}(u)}{\varphi(\zeta)-\varphi(u)}-\frac{1}{\zeta-u} .
$$

Proposition 5.1. If $\varphi$ is a full mapping, then the domination

$$
L_{\varphi, \Lambda_{0}, \Theta_{0}}(\zeta, u) \ll\left\{k_{\Lambda_{0}, \Theta_{0}}^{+}\left(\zeta, \zeta^{\prime}\right) ; \overline{k_{\Lambda_{0}, \Theta_{0}}^{-}\left(u, u^{\prime}\right)}\right\}
$$

is exact.

Proof. The proof of Theorem 5.2 shows that

$$
\left\|\sum_{k} c_{k} L_{\varphi, \Lambda_{0}, \Theta_{0}}\left(\cdot, u_{k}\right)\right\|_{\mathcal{D}_{\Lambda_{0}, 1-\Theta_{0}}}^{2}=\sum_{k, k^{\prime}} c_{k} \bar{c}_{k^{\prime}} k_{\Lambda_{0}, \Theta_{0}}^{-}\left(u_{k}, u_{k^{\prime}}\right),
$$

or equivalently,

$$
\left\|\sum_{k} c_{k} D^{\theta} L_{\varphi, \Lambda_{0}, \Theta_{0}}\left(\cdot, u_{k}\right)\right\|_{\mathcal{B}_{\Lambda_{0}, 1-\Theta_{0}}}=\sum_{k, k^{\prime}} c_{k} \bar{c}_{k^{\prime}} k_{\Lambda_{0}, \Theta_{0}}^{-}\left(u_{k}, u_{k^{\prime}}\right) .
$$

But an explicit calculation yields

$$
\begin{aligned}
& D_{\zeta}^{\theta} L_{\varphi, \Lambda_{0}, \Theta_{0}}(\zeta, u)=: M_{\varphi, \theta}(\zeta, u) \\
& \quad=-\left(\frac{\zeta}{\varphi(\zeta)}\right)^{1-\theta}\left(\frac{u}{\varphi(u)}\right)^{\theta} \frac{(1-\theta) \varphi(\zeta)+\theta \varphi(u)}{(\varphi(\zeta)-\varphi(u))^{2}} \varphi^{\prime}(\zeta) \varphi^{\prime}(u)+\frac{(1-\theta) \zeta+\theta u}{(\zeta-u)^{2}} .
\end{aligned}
$$

Rewriting (5.16) as

$$
\left\|\sum_{k} c_{k} M_{\varphi, \theta}\left(\cdot, u_{k}\right)\right\|_{\mathcal{B}_{\Lambda_{0}, 1-\Theta_{0}}}^{2}=\sum_{k, k^{\prime}} c_{k} \bar{c}_{k^{\prime}} k_{\Lambda_{0}, \Theta_{0}}^{-}\left(u_{k}, u_{k^{\prime}}\right)
$$

and using the symmetry property

$$
M_{\varphi, \theta}(\zeta, u)=M_{\varphi, 1-\theta}(u, \zeta),
$$

we obtain also

$$
\left\|\sum_{k} d_{k} M_{\varphi, \theta}\left(\zeta_{k}, \cdot\right)\right\|_{\mathcal{B}_{\Lambda_{0}, \Theta_{0}}}^{2}=\sum_{k, k^{\prime}} d_{k} \bar{d}_{k}^{\prime} k_{\Lambda_{0}, \mathbf{1}-\Theta_{0}}^{-}\left(\zeta_{k}, \zeta_{k}^{\prime}\right) .
$$

By Proposition 4.3, formulas (5.17) and (5.18) imply that we have an exact domination

$$
M_{\varphi, \theta}(\zeta, u) \ll\left\{k_{\Lambda_{0}, \mathbf{1}-\Theta_{0}}^{-}\left(\zeta, \zeta^{\prime}\right), \overline{k_{\Lambda_{0}, \Theta_{0}}^{-}\left(u, u^{\prime}\right)}\right\} .
$$

Since

$$
k_{\Lambda_{0}, \mathbf{1}-\Theta_{0}}^{-}\left(\zeta, \zeta^{\prime}\right)=D_{\zeta}^{\theta} D_{\bar{\zeta}^{\prime}}^{\theta} k_{\Lambda_{0}, \Theta_{0}}^{+}\left(\zeta, \zeta^{\prime}\right)
$$

by (5.14), we see that the domination (5.15) is exact by Proposition 4.4. 
We recall that the space $\mathcal{G}_{\theta}$ consists of $(\mathbb{T}, \theta)$-character-automorphic functions

$$
g(z)=\sum_{k \in \mathbb{Z}} \hat{g}(k) z^{k+\theta}
$$

such that

$$
\|g\|_{J, \theta}^{2}=\sum_{k \in \mathbb{Z}}|k+\theta||\hat{g}(k)|^{2}<+\infty .
$$

The closure of a subset of $\mathcal{G}_{\theta}$ means closure with respect to the Hilbert norm $\|\cdot\|_{J, \theta}$. Let $P_{\Lambda_{0}, \Theta_{0}}^{+}$(respectively, $P_{\Lambda_{0}, \Theta_{0}}^{-}$) denote the canonical projection to the positive (respectively, negative) subspace of the decomposition

$$
\mathcal{G}_{\theta}=\mathcal{G}_{\Lambda_{0}, \Theta_{0}}^{+} \dot{+}\left(\mathcal{G}_{\Lambda_{0}, \Theta_{0}}^{-}\right)^{\sharp} .
$$

For each $u \in \mathbb{D}$, let $g_{\varphi, \Lambda_{0}, \Theta_{0}, u} \in \mathcal{G}_{\theta}$ be defined by

$$
g_{\varphi, \Lambda_{0}, \Theta_{0}, u}(\zeta):=(\varphi(\zeta))^{\theta}\left(\frac{u}{\varphi(u)}\right)^{\theta} \frac{\varphi^{\prime}(u)}{\varphi(\zeta)-\varphi(u)} .
$$

Clearly, $g_{\varphi, \Lambda_{0}, \Theta_{0}, u}$ decomposes with respect to (5.19) as follows:

$$
g_{\varphi, \Lambda_{0}, \Theta_{0}, u}=g_{\varphi, \Lambda_{0}, \Theta_{0}, u}^{+}+g_{\varphi, \Lambda_{0}, \Theta_{0}, u}^{-},
$$

where

$$
g_{\varphi, \Lambda_{0}, \Theta_{0}, u}^{+}(\zeta)=\zeta^{\theta} L_{\varphi, \Lambda_{0}, \Theta_{0}}(\zeta, u)
$$

and

$$
g_{\varphi, \Lambda_{0}, \Theta_{0}, u}^{-}(\zeta)=\frac{\zeta^{\theta}}{\zeta-u}=\zeta^{\theta} f_{\Lambda_{0}, \Theta_{0}, u}(\zeta)
$$

If a function $g$ is a sum

$$
g=\sum_{k} c_{k} g_{\varphi, \Lambda_{0}, \Theta_{0}, u_{k}},
$$

and it decomposes with respect to (5.19) as

$$
g=P_{\Lambda_{0}, \Theta_{0}}^{+} g+P_{\Lambda_{0}, \Theta_{0}}^{-} g=g_{+}+g_{-},
$$

then by Corollary 2.2 we have

$$
\left[g_{+}, g_{+}\right]=-\left[g_{-}, g_{-}\right]=\frac{1}{2}\|g\|_{J, \theta}^{2} .
$$

Let $N_{\varphi, \Lambda_{0}, \Theta_{0}}$ denote the closure in $\mathcal{G}_{\theta}$ of the set of all functions $g$ given by (5.20). Clearly, $N_{\varphi, \Lambda_{0}, \Theta_{0}}$ is a closed isotropic subspace of $\mathcal{G}_{\theta}$; in particular, it is a positive subspace. The property that the linear combinations of the functions

$$
\zeta^{\theta} f_{\Lambda_{0}, \Theta_{0}, u}(\zeta)
$$

are dense in $\left(\mathcal{G}_{\Lambda_{0}, \Theta_{0}}^{-}\right)^{\sharp}$ (which is true by Lemma 3.6) together with (5.21) implies that

$$
P_{\Lambda_{0}, \Theta_{0}}^{-} N_{\varphi, \Lambda_{0}, \Theta_{0}}=\left(\mathcal{G}_{\Lambda_{0}, \Theta_{0}}^{-}\right)^{\sharp}
$$

(in other words, for any $g_{-} \in\left(\mathcal{G}_{\Lambda_{0}, \Theta_{0}}^{-}\right)^{\sharp}$ there exists $g \in N_{\varphi, \Lambda_{0}, \Theta_{0}}$ with $g_{-}=P_{\Lambda_{0}, \Theta_{0}}^{-} g$ ). Similarly, since the functions

$$
g_{\varphi, \Lambda_{0}, \Theta_{0}, u}^{+}(\zeta)=\zeta^{\theta} L_{\varphi, \Lambda_{0}, \Theta_{0}}(\zeta, u)
$$

form a complete system in $\mathcal{G}_{\Lambda_{0}, \Theta_{0}}^{+}$(because the domination (5.15) is exact), we have

$$
P_{\Lambda_{0}, \Theta_{0}}^{+} N_{\varphi, \Lambda_{0}, \Theta_{0}}=\mathcal{G}_{\Lambda_{0}, \Theta_{0}}^{+} .
$$

Lemma 5.1. $N_{\varphi, \Lambda_{0}, \Theta_{0}}$ is a maximal positive subspace of $\mathcal{G}_{\theta}$. 
Proof. By Lemma V.4.5 in [3, it suffices to show that the orthogonal companion to $N_{\varphi, \Lambda_{0}, \Theta_{0}}$ is negative. Let $h \in \mathcal{G}_{\theta}$ be orthogonal to $N_{\varphi, \Lambda_{0}, \Theta_{0}}$, and let

$$
h=P_{\Lambda_{0}, \Theta_{0}}^{+} h+P_{\Lambda_{0}, \Theta_{0}}^{-} h=h_{+}+h_{-} .
$$

By the above remarks, there exists $g \in N_{\varphi, \Lambda_{0}, \Theta_{0}}$ with $g_{+}=P_{\Lambda_{0}, \Theta_{0}}^{+} h=h_{+}$. Then

$$
0=[g, h]=\left[g_{+}, g_{+}\right]+\left[g_{-}, h_{-}\right] .
$$

Since

$$
\left[g_{+}, g_{+}\right]^{2}=\left|\left[g_{-}, h_{-}\right]\right|^{2} \leq\left[g_{-}, g_{-}\right] \cdot\left[h_{-}, h_{-}\right]=-\left[g_{+}, g_{+}\right] \cdot\left[h_{-}, h_{-}\right],
$$

we obtain

$$
-\left[h_{-}, h_{-}\right] \geq\left[g_{+}, g_{+}\right]=\left[h_{+}, h_{+}\right],
$$

showing that

$$
[h, h] \leq 0
$$

Remark. The same arguments also show that $N_{\varphi, \Lambda_{0}, \Theta_{0}}$ is a maximal negative subspace of $\mathcal{G}_{\theta}$. It is easily seen that the orthogonal companion to $N_{\varphi, \Lambda_{0}, \Theta_{0}}$ coincides with $N_{\varphi, \Lambda_{0}, \Theta_{0}}$ itself.

We turn to the case of general $\Lambda=\left\{\lambda_{1}, \ldots, \lambda_{n}\right\}$ and $\Theta=\left\{\theta_{1}, \ldots, \theta_{n}\right\}$. As before, we assume that $\theta=\theta_{1}+\cdots+\theta_{n} \leq 1$. Now, the symbols $P_{\Lambda, \Theta}^{+}$and $P_{\Lambda, \Theta}^{-}$stand for the canonical projections to the positive and the negative subspace of the decomposition (3.29), and the functions $g_{\varphi, \Lambda, \Theta, u}$ are defined by

$$
\begin{aligned}
g_{\varphi, \Lambda, \Theta, u}(\zeta):= & {\left[\prod_{k=1}^{n}\left(\varphi(\zeta)-\varphi\left(\lambda_{k}\right)\right)^{\theta_{k}}\right] } \\
& \times\left[\prod_{k=1}^{n}\left(\frac{u-\lambda_{k}}{\left(1-\bar{\lambda}_{k} u\right)\left(\varphi(u)-\varphi\left(\lambda_{k}\right)\right)}\right)^{\theta_{k}}\right] \cdot \frac{\varphi^{\prime}(u)}{\varphi(\zeta)-\varphi(u)},
\end{aligned}
$$

so that

$$
P_{\Lambda, \Theta}^{+} g_{\varphi, \Lambda, \Theta, u}(\zeta)=\omega_{\Lambda, \Theta}(\zeta) L_{\varphi, \Lambda, \Theta}(\zeta, u)
$$

and

$$
P_{\Lambda, \Theta}^{-} g_{\varphi, \Lambda, \Theta, u}(\zeta)=\omega_{\Lambda, \Theta}(\zeta) f_{\Lambda, \Theta, u}(\zeta)
$$

Let $N_{\varphi, \Lambda, \Theta}$ be the closure in $\mathcal{G}_{\theta}$ of the linear combinations of the functions $g_{\varphi, \Lambda, \Theta, u}$.

Lemma 5.2. $N_{\varphi, \Lambda_{0}, \Theta_{0}}=N_{\varphi, \Lambda, \Theta}$.

Proof. Let $u$ be some fixed point of $\mathbb{D}$, and let $\Omega$ be a subdomain of $\mathbb{D}$ such that $\left\{u, \lambda_{1}, \ldots, \lambda_{n}\right\} \in \Omega$ and $\bar{\Omega} \subset \mathbb{D}$. We introduce the function

$$
F(v)=\prod_{k=1}^{n}\left(\frac{v-\varphi\left(\lambda_{k}\right)}{v}\right)^{\theta_{k}} \cdot \frac{1}{v-\varphi(u)},
$$

choosing some branch of it that is regular and single-valued in $\overline{\mathbb{C}} \backslash \varphi(\bar{\Omega})$. Let $\gamma$ be some simple positively oriented contour in $\mathbb{D}$ such that $\bar{\Omega} \subset$ int $\gamma$. Then we have

$$
-\frac{1}{2 \pi i} \int_{\varphi(\gamma)} \frac{F(v)}{v-\varphi(\zeta)} d v=\left[\prod_{k=1}^{n}\left(\frac{\varphi(\zeta)-\varphi\left(\lambda_{k}\right)}{\varphi(\zeta)}\right)^{\theta_{k}}\right] \cdot \frac{1}{\varphi(\zeta)-\varphi(u)}
$$


if $\zeta$ is near $\mathbb{T}$, which implies that

$$
\begin{aligned}
& -\frac{1}{2 \pi i} \int_{\gamma} F(\varphi(\eta))(\varphi(\zeta))^{\theta} \frac{\varphi^{\prime}(\eta)}{\varphi(\eta)-\varphi(\zeta)} d \eta \\
& \quad=\left[\prod_{k=1}^{n}\left(\varphi(\zeta)-\varphi\left(\lambda_{k}\right)\right)^{\theta_{k}}\right] \cdot \frac{1}{\varphi(\zeta)-\varphi(u)}
\end{aligned}
$$

for $\zeta$ near $\mathbb{T}$. Therefore, $N_{\varphi, \Lambda, \Theta} \subset N_{\varphi, \Lambda_{0}, \Theta_{0}}$. The reverse inclusion is proved similarly.

We are ready to prove the following statement.

Theorem 5.3. If $\varphi$ is a full mapping, then the domination (5.8) is exact.

Proof. It suffices to show that the functions $L_{\varphi, \Lambda, \Theta}(\cdot, u)$ form a complete system in $\mathcal{D}_{\Lambda, \Theta}$, or equivalently, the functions $\omega_{\Lambda, \Theta}(\cdot) L_{\varphi, \Lambda, \Theta}(\cdot, u)$ form a complete system in $\mathcal{G}_{\Lambda, \Theta}^{+}$. Let $h \in \mathcal{G}_{\Lambda, \Theta}^{+}$be a function orthogonal to all functions $\omega_{\Lambda, \Theta}(\cdot) L_{\varphi, \Lambda, \Theta}(\cdot, u)$. Then, by (5.22), $h$ is orthogonal to all functions $g_{\varphi, \Lambda, \Theta, u}$, so that $h$ lies in the orthogonal complement to the subspace $N_{\varphi, \Lambda, \Theta}$. Since $N_{\varphi, \Lambda, \Theta}=N_{\varphi, \Lambda_{0}, \Theta_{0}}$ is a maximal positive subspace of $\mathcal{G}_{\theta}$, we see that

whence $h=0$.

$$
[h, h] \leq 0
$$

In the context of Theorem 5.1 the domination (5.1) is not exact in the case where $\psi$ is a full self-map of $\mathbb{D}$. Indeed, if $p$ is an analytic polynomial, then

$$
\begin{aligned}
& \left(l_{\psi, \Lambda, \Theta}(\cdot, u), \frac{\omega_{\psi(\Lambda), \Theta}(\psi(\cdot))}{\omega_{\Lambda, \Theta}(\cdot)} p(\psi(\cdot))\right)_{\mathcal{D}_{\Lambda, \Theta}} \\
& \quad=\left[\omega_{\psi(\Lambda), \Theta}(\psi(\cdot)) \frac{\omega_{\Lambda, \Theta}(u)}{\omega_{\psi(\Lambda), \Theta}(\psi(u))} \psi^{\prime}(u) \cdot f_{\psi(\Lambda), \Theta, \psi(u)}(\psi(\cdot)), \omega_{\psi(\Lambda), \Theta}(\psi(\cdot)) p(\psi(\cdot))\right] \\
& \quad=\left[\omega_{\psi(\Lambda), \Theta}(\cdot) \frac{\omega_{\Lambda, \Theta}(u)}{\omega_{\psi(\Lambda), \Theta}(\psi(u))} \psi^{\prime}(u) \cdot f_{\psi(\Lambda), \Theta, \psi(u)}(\cdot), \omega_{\psi(\Lambda), \Theta}(\cdot) p(\cdot)\right]=0,
\end{aligned}
$$

which shows that the functions $l_{\psi, \Lambda, \Theta}(\cdot, u)$ cannot form a complete system in $\mathcal{D}_{\Lambda, \Theta}$. Apparently, the exact domination in this context is

$$
l_{\psi, \Lambda, \Theta}(\zeta, u) \ll\left\{k_{\Lambda, \Theta}^{+}\left(\zeta, \zeta^{\prime}\right)-k_{\psi, \Lambda, \Theta}^{+}\left(\zeta, \zeta^{\prime}\right) ; \overline{k_{\Lambda, \Theta}^{-}\left(u, u^{\prime}\right)-k_{\psi, \Lambda, \Theta}^{-}\left(u, u^{\prime}\right)}\right\},
$$

where

$$
k_{\psi, \Lambda, \Theta}^{+}\left(\zeta, \zeta^{\prime}\right)=\frac{\omega_{\psi(\Lambda), \Theta}(\psi(\zeta))}{\omega_{\Lambda, \Theta}(\zeta)} \overline{\left(\frac{\omega_{\psi(\Lambda), \Theta}\left(\psi\left(\zeta^{\prime}\right)\right)}{\omega_{\Lambda, \Theta}\left(\zeta^{\prime}\right)}\right)} k_{\Lambda, \Theta}^{+}\left(\psi\left(\zeta, \psi\left(\zeta^{\prime}\right)\right)\right)
$$

and $k_{\psi, \Lambda, \Theta}^{-}\left(u, u^{\prime}\right)$ is defined by (5.2).

\section{$\S 6$. Special CASES}

In this section we consider special cases of one or two branching points and obtain explicit integral inequalities as special cases of the main Theorem 5.2 .

One branching point. First, let $\Lambda$ consist of a single point $\lambda \in \mathbb{D}$, and let $\theta \in(0,1)$. Then the norms $\|\cdot\|_{\mathcal{D}_{\Lambda, \Theta}}$ and $\|\cdot\|_{\mathcal{B}_{\Lambda, \Theta}}$ look like this:

$$
\|g\|_{\mathcal{D}_{\Lambda, \Theta}}^{2}=\int_{\mathbb{D}}\left|\theta \frac{1-|\lambda|^{2}}{(1-\bar{\lambda} z)^{2}} g(z)+\omega_{\lambda}(z) g^{\prime}(z)\right|^{2} \frac{d A(z)}{\left|\omega_{\lambda}(z)\right|^{2-2 \theta}}
$$

and

$$
\|x\|_{\mathcal{B}_{\Lambda, \Theta}}^{2}=\int_{\mathbb{D}}|x(u)|^{2} \frac{d A(u)}{\left|\omega_{\lambda}(u)\right|^{2 \theta}}
$$


Lemma 6.1. Suppose $\Lambda=\{\lambda\}$ and $\Theta=\{\theta\}$. Then

$$
k_{\Lambda, \Theta}^{+}\left(z, z^{\prime}\right)=\sum_{n \geq 0} \frac{\left(\omega_{\lambda}(z) \overline{\omega_{\lambda}\left(z^{\prime}\right)}\right)^{n}}{n+\theta}
$$

and

$$
\begin{aligned}
k_{\Lambda, \Theta}^{-}\left(u, u^{\prime}\right) & =\frac{\left(1-|\lambda|^{2}\right)^{2}}{(1-\bar{\lambda} u)^{2}\left(1-\lambda \bar{u}^{\prime}\right)^{2}} \sum_{n \geq 0}(n+1-\theta)\left(\omega_{\lambda}(u) \overline{\omega_{\lambda}\left(u^{\prime}\right)}\right)^{n} \\
& =\frac{1}{\left(1-u \bar{u}^{\prime}\right)^{2}}-\frac{\theta\left(1-|\lambda|^{2}\right)}{(1-\bar{\lambda} u)\left(1-\lambda \bar{u}^{\prime}\right)\left(1-u \bar{u}^{\prime}\right)} .
\end{aligned}
$$

Proof. For $\lambda=0$, formula (6.2) was obtained in 95 . On the other hand, the identity

$$
\left[\omega_{\lambda}^{\theta} f, \omega_{\lambda}^{\theta} g\right]=\left[z^{\theta}\left(f \circ \omega_{-\lambda}\right), z^{\theta}\left(g \circ \omega_{-\lambda}\right)\right]
$$

shows that

$$
k_{\{\lambda\},\{\theta\}}^{+}\left(z, z^{\prime}\right)=k_{\{0\},\{\theta\}}^{+}\left(\omega_{\lambda}(z), \omega_{\lambda}\left(z^{\prime}\right)\right)
$$

which gives (6.2).

Similarly, formula (6.3) for $\lambda=0$ was obtained in $\$ 5$ (see (5.14)). The identity

$$
(f, g)_{\mathcal{B}_{\{\lambda\},\{\theta\}}}=\left(\left(f \circ \omega_{-\lambda}\right) \omega_{-\lambda}^{\prime},\left(g \circ \omega_{-\lambda}\right) \omega_{-\lambda}^{\prime}\right)_{\mathcal{B}_{\{0\},\{\theta\}}}
$$

implies that

$$
k_{\{\lambda\},\{\theta\}}^{-}\left(u, u^{\prime}\right)=\omega_{\lambda}^{\prime}(u) \overline{\omega_{\lambda}^{\prime}\left(u^{\prime}\right)} k_{\{0\},\{\theta\}}^{-}\left(\omega_{\lambda}(u), \omega_{\lambda}\left(u^{\prime}\right)\right),
$$

which gives (6.3).

Combining Theorems 5.2 and 4.1, we obtain the following statement.

Corollary 6.1. Let $\varphi$ be univalent in $\mathbb{D}$, and let $\zeta_{1}, \ldots, \zeta_{n} \in \mathbb{D}$ and $\alpha_{1}, \ldots, \alpha_{n} \in \mathbb{C}$. Then

$$
\int_{\mathbb{D}}\left|\sum_{\nu=1}^{N} \alpha_{\nu} L_{\varphi}\left(\zeta_{\nu}, u\right)\right|^{2} \frac{d A(u)}{\left|\omega_{\lambda}(u)\right|^{2 \theta}} \leq \sum_{1 \leq \nu, \nu^{\prime} \leq N} \alpha_{\nu} \bar{\alpha}_{\nu^{\prime}} k_{\Lambda, \Theta}^{+}\left(\zeta_{\nu}, \zeta_{\nu^{\prime}}\right),
$$

where $k_{\Lambda, \Theta}^{+}$is given by (6.2) and

$$
L_{\varphi}(\zeta, u)=\left(\frac{\varphi(\zeta)-\varphi(\lambda)}{\zeta-\lambda} \frac{u-\lambda}{\varphi(u)-\varphi(\lambda)} \frac{1-\bar{\lambda} \zeta}{1-\bar{\lambda} u}\right)^{\theta} \frac{\varphi^{\prime}(u)}{\varphi(\zeta)-\varphi(u)}-\frac{1}{\zeta-u}+\frac{\bar{\lambda}}{1-\bar{\lambda} u} .
$$

Moreover, equality occurs in (6.4) if $\varphi$ is a full mapping.

If $N=1$ and $\zeta_{1}=\lambda$, inequality (6.4) reduces to

$$
\begin{aligned}
\int_{\mathbb{D}}\left|\left(\varphi^{\prime}(\lambda) \frac{u-\lambda}{\varphi(u)-\varphi(\lambda)} \frac{1-|\lambda|^{2}}{1-\bar{\lambda} u}\right)^{\theta} \frac{\varphi^{\prime}(u)}{\varphi(\lambda)-\varphi(u)}-\frac{1}{\lambda-u}+\frac{\bar{\lambda}}{1-\bar{\lambda} u}\right|^{2} \frac{d A(u)}{\left|\omega_{\lambda}(u)\right|^{2 \theta}} \\
\quad \leq \frac{1}{\theta} .
\end{aligned}
$$

This integral inequality can also be obtained directly from Prawitz' inequality (1.5) by appropriate Möbius transformations; see [7. In that paper, inequality (6.5) (written in a slightly different form) was a starting point for deriving estimates of the integral means of derivatives of univalent functions. 
Two branching points. Now, we turn to the calculations in the case of two branching points. In the remaining part of this section, we assume that $\Lambda=\left\{0, \lambda_{0}\right\}$ and $\Theta=\left\{\theta_{1}, \theta_{2}\right\}$ with $\theta_{1}, \theta_{2}>0, \theta_{1}+\theta_{2} \leq 1$. The case of general $\Lambda=\left\{\lambda_{1}, \lambda_{2}\right\}$ can be reduced to $\Lambda=\left\{0, \lambda_{0}\right\}$ by an appropriate Möbius shift of variables.

We start with explicit formulas for the norms $\|\cdot\|_{\mathcal{D}_{\Lambda, \Theta}}$ and $\|\cdot\|_{\mathcal{B}_{\Lambda, \Theta}}$. We have

$$
\begin{aligned}
\|g\|_{\mathcal{D}_{\Lambda, \Theta}}^{2}= & \int_{\mathbb{D}}\left|\left(\theta_{1} \omega_{\lambda_{0}}(z)+\theta_{2} z \frac{1-\left|\lambda_{0}\right|^{2}}{\left(1-\bar{\lambda}_{0} z\right)^{2}}\right) g(z)+z \omega_{\lambda_{0}}(z) g^{\prime}(z)\right|^{2} \\
& \times \frac{d A(z)}{|z|^{2-2 \theta_{1}}\left|\omega_{\lambda_{0}}(z)\right|^{2-2 \theta_{2}}}
\end{aligned}
$$

and

$$
\|x\|_{\mathcal{B}_{\Lambda, \Theta}}^{2}=\int_{\mathbb{D}}|x(u)|^{2} \frac{d A(u)}{|u|^{2 \theta_{1}}\left|\omega_{\lambda_{0}}(u)\right|^{2 \theta_{2}}}+\frac{\sin \pi \theta_{1} \cdot \sin \pi \theta_{2}}{\pi \sin \left(\pi\left(\theta_{1}+\theta_{2}\right)\right)}\left|\int_{0}^{\lambda_{0}} \frac{x(u)}{u^{\theta_{1}} \omega_{\lambda_{0}} \theta_{2}(u)} d u\right|^{2}
$$

if $\theta_{1}+\theta_{2}<1$, and

$$
\|x\|_{\mathcal{B}_{\Lambda, \Theta}}^{2}=\int_{\mathbb{D}}|x(u)|^{2} \frac{d A(u)}{|u|^{2 \theta_{1}}\left|\omega_{\lambda_{0}}(u)\right|^{2 \theta_{2}}}
$$

if $\theta_{1}+\theta_{2}=1$; in the latter case $\mathcal{B}_{\Lambda, \Theta}$ consists of all functions $x \in \mathcal{B}_{0}^{+}$that satisfy

$$
\int_{0}^{\lambda_{0}} \frac{x(u)}{u^{\theta_{1}} \omega_{\lambda_{0}} \theta_{2}(u)} d u=0
$$

The next lemma shows that the kernel $k_{\Lambda, \Theta}^{+}(z, \mu)$ is a solution of a certain first order differential equation.

Lemma 6.2. We have

$$
z \partial_{z}\left(\omega_{\Lambda, \Theta}(z) k_{\Lambda, \Theta}^{+}(z, \mu)\right)=\omega_{\Lambda, \Theta}(z)\left(\frac{1}{1-\bar{\mu} z}+\frac{\theta_{2} \lambda_{0}}{z-\lambda_{0}} k_{\Lambda, \Theta}^{+}\left(\lambda_{0}, \mu\right)\right) .
$$

Proof. For any polynomial $p$, we can write

$$
\begin{aligned}
\overline{p(\mu)} & =\left[\omega_{\Lambda, \Theta}(\cdot) k_{\Lambda, \Theta}^{+}(\cdot, \mu), \omega_{\Lambda, \Theta}(\cdot) p(\cdot)\right] \\
& =\int_{\mathbb{T}} z \partial_{z}\left(\omega_{\Lambda, \Theta}(z) k_{\Lambda, \Theta}^{+}(z, \mu)\right) \overline{\omega_{\Lambda, \Theta}(z) p(z)} d m(z),
\end{aligned}
$$

which shows that

$$
\overline{\omega_{\Lambda, \Theta}(z)} z \partial_{z}\left(\omega_{\Lambda, \Theta}(z) k_{\Lambda, \Theta}^{+}(z, \mu)\right)=\frac{1}{1-\bar{\mu} z}+\overline{z r(z)}, \quad z \in \mathbb{T},
$$

where $r(\cdot)$ is some function in $\mathcal{B}_{0}^{+}$. On the other hand, for $z \in \mathbb{D}$ we have

$$
\begin{aligned}
& \frac{1}{\omega_{\Lambda, \Theta}(z)} z \partial_{z}\left(\omega_{\Lambda, \Theta}(z) k_{\Lambda, \Theta}^{+}(z, \mu)\right) \\
& \quad=\left(\theta_{1}+\theta_{2} z \frac{1-\left|\lambda_{0}\right|^{2}}{\left(z-\lambda_{0}\right)\left(1-\bar{\lambda}_{0} z\right)}\right) k_{\Lambda, \Theta}^{+}(z, \mu)+z \partial_{z} k_{\Lambda, \Theta}^{+}(z, \mu) \\
& \quad=\theta_{2} \frac{\lambda_{0} k_{\Lambda, \Theta}^{+}\left(\lambda_{0}, \mu\right)}{z-\lambda_{0}}+h(z),
\end{aligned}
$$

where $h \in \mathcal{B}_{0}^{+}$. Comparing (6.10) and (6.11), we obtain

$$
\overline{z r(z)}=\theta_{2} \frac{\lambda_{0} k_{\Lambda, \Theta}^{+}\left(\lambda_{0}, \mu\right)}{z-\lambda_{0}}, \quad z \in \mathbb{T},
$$

which proves (6.8). 
Remark. Similar arguments show that in the case of general $\Lambda=\left\{\lambda_{1}, \ldots, \lambda_{n}\right\}$ and $\Theta=$ $\left\{\theta_{1}, \ldots, \theta_{n}\right\}$ we have

$$
z \partial_{z}\left(\omega_{\Lambda, \Theta}(z) k_{\Lambda, \Theta}^{+}(z, \mu)\right)=\omega_{\Lambda, \Theta}(z)\left(\frac{1}{1-\bar{\mu} z}+\sum_{k=1}^{n} \frac{\theta_{k} \lambda_{k}}{z-\lambda_{k}} k_{\Lambda, \Theta}^{+}\left(\lambda_{k}, \mu\right)\right) .
$$

We return to the special case of $\Lambda=\left\{0, \lambda_{0}\right\}, \Theta=\left\{\theta_{1}, \theta_{2}\right\}$. The kernels $k_{\Lambda, \Theta}^{+}(\cdot, 0)$ and $k_{\Lambda, \Theta}^{+}\left(\cdot, \lambda_{0}\right)$ can be described by alternative formulas. First, we have the following statement.

\section{Lemma 6.3.}

$$
\theta_{1} k_{\Lambda, \Theta}^{+}(\mu, 0)+\theta_{2} k_{\Lambda, \Theta}^{+}\left(\mu, \lambda_{0}\right)=1, \quad \mu \in \mathbb{D} .
$$

Proof. We have

$$
\begin{aligned}
1 & =\left[z^{\theta_{1}} \omega_{\lambda_{0}}^{\theta_{2}}(z) \cdot 1, z^{\theta_{1}} \omega_{\lambda_{0}}^{\theta_{2}}(z) k_{\Lambda, \Theta}^{+}(z, \mu)\right] \\
& =\int_{\mathbb{T}} z \partial_{z}\left(z^{\theta_{1}} \omega_{\lambda_{0}}^{\theta_{2}}(z)\right) \overline{z^{\theta_{1}} \omega_{\lambda_{0}}^{\theta_{2}}(z) k_{\Lambda, \Theta}^{+}(z, \mu)} d m(z) .
\end{aligned}
$$

Since

we obtain

$$
z \partial_{z}\left(z^{\theta_{1}} \omega_{\lambda_{0}}^{\theta_{2}}(z)\right)=z^{\theta_{1}} \omega_{\lambda_{0}}^{\theta_{2}}(z)\left(\theta_{1}+\theta_{2} \frac{1-\left|\lambda_{0}\right|^{2}}{\left|1-\bar{\lambda}_{0} z\right|^{2}}\right), \quad z \in \mathbb{T}
$$

$$
1=\int_{\mathbb{T}}\left(\theta_{1}+\theta_{2} \frac{1-\left|\lambda_{0}\right|^{2}}{\left|1-\bar{\lambda}_{0} z\right|^{2}}\right) \overline{k_{\Lambda, \Theta}^{+}(z, \mu)} d m(z)=\theta_{1} k_{\Lambda, \Theta}^{+}(0, \mu)+\theta_{2} k_{\Lambda, \Theta}^{+}\left(\lambda_{0}, \mu\right)
$$

which implies (6.12).

Our next goal is to describe the kernel $k_{\Lambda, \Theta}^{+}(\cdot, 0)$. We introduce the function

$$
X_{0}(z)=z+\bar{\lambda}_{0} \theta_{1} z \omega_{\lambda}(z) k_{\Lambda, \Theta}^{+}(z, 0) \text {; }
$$

then

$$
k_{\Lambda, \Theta}^{+}(z, 0)=\frac{X_{0}(z)-z}{\bar{\lambda}_{0} \theta_{1} z \omega_{\lambda_{0}}(z)} .
$$

Lemma 6.4. The function $z^{\theta_{1}-1} \omega_{\lambda}(z)^{\theta_{2}-1} X_{0}(z)$ is orthogonal (relative to the Dirichlet form) to $\mathcal{G}_{\Lambda, \Theta}^{+}$.

Proof. For any polynomial $p$, we can write

$$
\begin{aligned}
{\left[z^{\theta_{1}-1} \omega_{\lambda}(z)^{\theta_{2}-1} X_{0}(z), z^{\theta_{1}} \omega_{\lambda}^{\theta_{2}}(z) p(z)\right] } & =\left[z^{\theta_{1}} \omega_{\lambda}(z)^{\theta_{2}-1}, z^{\theta_{1}} \omega_{\lambda}(z)^{\theta_{2}-1} \cdot \omega_{\lambda}(z) p(z)\right] \\
& +\bar{\lambda}_{0} \theta_{1}\left[z^{\theta_{1}} \omega_{\lambda}^{\theta_{2}}(z) k_{\Lambda, \Theta}^{+}(z, 0), z^{\theta_{1}} \omega_{\lambda}^{\theta_{2}}(z) p(z)\right] \\
= & \int_{\mathbb{T}}\left(\theta_{1}+\left(\theta_{2}-1\right) \frac{1-\left|\lambda_{0}\right|^{2}}{\left|1-\bar{\lambda}_{0} \zeta\right|^{2}}\right) \overline{\omega_{\lambda}(\zeta) p(\zeta)} d m(\zeta)+\bar{\lambda}_{0} \theta_{1} \overline{p(0)} \\
= & -\bar{\lambda}_{0} \theta_{1} \overline{p(0)}+\bar{\lambda}_{0} \theta_{1} \overline{p(0)}=0 .
\end{aligned}
$$

For any $a \in \mathbb{R}$, let $(a)_{k}$ stand for the Pochhammer symbol

$$
(a)_{k}:=a(a+1) \cdots \cdot(a+k-1) .
$$

We denote by $\mathcal{S}$ the shift operator,

$$
(\mathcal{S} f)(z):=z f(z)
$$


and by $\mathcal{L}$ the left inverse to $\mathcal{S}$, i.e., the backward shift operator,

$$
(\mathcal{L} g)(z):=\frac{g(z)-g(0)}{z}
$$

(both operators are defined on functions analytic in $\mathbb{D}$ ). Then, for any $h$ analytic in $\overline{\mathbb{D}}$, we have

$$
\int_{\mathbb{T}} h(\zeta) f(\zeta) \overline{g(\zeta)} d m(\zeta)=\int_{\mathbb{T}} f(\zeta) \overline{\left[h_{*}(\mathcal{L}) g\right](\zeta)} d m(\zeta)
$$

(in all situations where both sides of this identity make sense), where

$$
h_{*}(z)=\overline{h(\bar{z})} .
$$

Proposition 6.1. The function $X_{0}$ defined by (6.13) admits the following representation:

$$
\begin{aligned}
X_{0}(z) & =a_{0} z\left(I-\lambda_{0} \mathcal{L}\right)^{1-\theta_{2}}\left[\sum_{k \geq 0} \frac{\left(\theta_{1}\right)_{k}}{\left(\theta_{1}+\theta_{2}\right)_{k}} \bar{\lambda}_{0}^{k} z^{k}\right] \\
& =a_{0} z \sum_{k \geq 0}\left\{\sum_{l \geq 0} \frac{\left(\theta_{2}-1\right)_{l}\left(\theta_{1}\right)_{k+l}}{l !\left(\theta_{1}+\theta_{2}\right)_{k+l}}\left|\lambda_{0}\right|^{2 l}\right\} \bar{\lambda}_{0}^{k} z^{k},
\end{aligned}
$$

where the constant $a_{0}$ is chosen so that $X_{0}\left(\lambda_{0}\right)=\lambda_{0}$.

Proof. Lemma 6.4 implies that

$$
\left[z^{\theta_{1}}\left(z-\lambda_{0}\right)^{\theta_{2}} p(z), z^{\theta_{1}-1} \omega_{\lambda_{0}}(z)^{\theta_{2}-1} X_{0}(z)\right]=0
$$

for any polynomial $p$. This identity can be written as

$$
\begin{aligned}
0= & \int_{\mathbb{T}}\left[\left(\theta_{1}\left(z-\lambda_{0}\right)+\theta_{2} z\right) p(z)+z\left(z-\lambda_{0}\right) \partial_{z} p(z)\right] \\
& \times z^{\theta_{1}-1}\left(z-\lambda_{0}\right)^{\theta_{2}-1} \cdot \overline{z^{\theta_{1}-1} \omega_{\lambda_{0}}(z)^{\theta_{2}-1} X_{0}(z)} z d m(z) \\
= & \int_{\mathbb{T}}\left[\left(\left(\theta_{1}+\theta_{2}\right) z-\theta_{1} \lambda_{0}\right) p(z)+\left(z-\lambda_{0}\right) z \partial_{z} p(z)\right]\left(1-\bar{\lambda}_{0} z\right)^{\theta_{2}-1} z \overline{X_{0}(z)} d m(z) \\
= & \int_{\mathbb{T}} p(z) \cdot \overline{\left[\left(\theta_{1}+\theta_{2}\right) \mathcal{L}-\theta_{1} \bar{\lambda}_{0}+z \partial_{z}\left(\mathcal{L}-\bar{\lambda}_{0} I\right)\right]\left(I-\lambda_{0} \mathcal{L}\right)^{\theta_{2}-1} \mathcal{L} X_{0}(z)} d m(z),
\end{aligned}
$$

which gives

$$
\left[\left(\theta_{1}+\theta_{2}\right) \mathcal{L}-\theta_{1} \bar{\lambda}_{0}+z \partial_{z}\left(\mathcal{L}-\bar{\lambda}_{0} I\right)\right]\left(I-\lambda_{0} \mathcal{L}\right)^{\theta_{2}-1} \mathcal{L} X_{0}=0
$$

Let $Y_{0}=\left(I-\lambda_{0} \mathcal{L}\right)^{\theta_{2}-1} \mathcal{L} X_{0}$. Since $X_{0}(0)=0$, we have

$$
X_{0}(z)=z\left[\left(I-\lambda_{0} \mathcal{L}\right)^{1-\theta_{2}} Y_{0}\right](z) .
$$

If

$$
Y_{0}(z)=\sum_{k \geq 0} a_{k} z^{k}
$$

then (6.17) is equivalent to

$$
\left[\left(\theta_{1}+\theta_{2}\right) \mathcal{L}-\theta_{1} \bar{\lambda}_{0}+z \partial_{z}\left(\mathcal{L}-\bar{\lambda}_{0} I\right)\right] Y_{0}=0,
$$

or

$$
\left(\theta_{1}+\theta_{2}+k\right) a_{k+1}-\left(\theta_{1}+k\right) \bar{\lambda}_{0} a_{k}=0,
$$

which yields

$$
a_{k}=\frac{\left(\theta_{1}\right)_{k} \bar{\lambda}_{0}^{k}}{\left(\theta_{1}+\theta_{2}\right)_{k}} a_{0}
$$

and implies (6.16). The normalization $X_{0}\left(\lambda_{0}\right)=\lambda_{0}$ follows from the definition (6.13). 
The right-hand side of (6.16) admits an integral representation. Namely, since

$$
(a)_{k}=\frac{\Gamma(a+k)}{\Gamma(a)}
$$

the standard properties of Euler integrals imply

$$
\frac{\left(\theta_{1}\right)_{k}}{\left(\theta_{1}+\theta_{2}\right)_{k}}=\frac{\Gamma\left(\theta_{1}+k\right) \Gamma\left(\theta_{1}+\theta_{2}\right)}{\Gamma\left(\theta_{1}\right) \Gamma\left(\theta_{1}+\theta_{2}+k\right)}=\frac{\Gamma\left(\theta_{1}+\theta_{2}\right)}{\Gamma\left(\theta_{1}\right) \Gamma\left(\theta_{2}\right)} \int_{0}^{1} t^{\theta_{1}-1+k}(1-t)^{\theta_{2}-1} d t .
$$

Therefore,

$$
\sum_{k \geq 0} \frac{\left(\theta_{1}\right)_{k}}{\left(\theta_{1}+\theta_{2}\right)_{k}}\left(\bar{\lambda}_{0} z\right)^{k}=\frac{\Gamma\left(\theta_{1}+\theta_{2}\right)}{\Gamma\left(\theta_{1}\right) \Gamma\left(\theta_{2}\right)} \int_{0}^{1} \frac{t^{\theta_{1}-1}(1-t)^{\theta_{2}-1}}{1-\bar{\lambda}_{0} z t} d t
$$

Since

we obtain

$$
\mathcal{L}\left[\frac{1}{1-\bar{\lambda}_{0} z t}\right]=\frac{\bar{\lambda}_{0} t}{1-\bar{\lambda}_{0} z t}
$$

$$
X_{0}(z)=a_{0} z \frac{\Gamma\left(\theta_{1}+\theta_{2}\right)}{\Gamma\left(\theta_{1}\right) \Gamma\left(\theta_{2}\right)} \int_{0}^{1} \frac{\left(1-\left|\lambda_{0}\right|^{2} t\right)^{1-\theta_{2}} t^{\theta_{1}-1}(1-t)^{\theta_{2}-1}}{1-\bar{\lambda}_{0} z t} d t .
$$

Let $E_{\theta_{1}, \theta_{2}}$ denote the integral

$$
E_{\theta_{1}, \theta_{2}}(z):=\int_{0}^{1} \frac{\left(1-\left|\lambda_{0}\right|^{2} t\right)^{1-\theta_{2}} t^{\theta_{1}-1}(1-t)^{\theta_{2}-1}}{1-\bar{\lambda}_{0} z t} d t
$$

(depending also on $\lambda_{0}$ ). Finally, we get

$$
X_{0}(z)=z E_{\theta_{1}, \theta_{2}}^{-1}\left(\lambda_{0}\right) E_{\theta_{1}, \theta_{2}}(z)
$$

and

$$
k_{\Lambda, \Theta}^{+}(z, 0)=\frac{E_{\theta_{1}, \theta_{2}}^{-1}\left(\lambda_{0}\right) E_{\theta_{1}, \theta_{2}}(z)-1}{\bar{\lambda}_{0} \theta_{1} \omega_{\lambda_{0}}(z)} .
$$

Combining this formula with (6.12), we can also find an integral representation for $k_{\Lambda, \Theta}^{+}\left(z, \lambda_{0}\right)$ :

$$
k_{\Lambda, \Theta}^{+}\left(z, \lambda_{0}\right)=\frac{\frac{1-\left|\lambda_{0}\right|^{2}}{1-\lambda_{0} z}-E_{\theta_{1}, \theta_{2}}^{-1}\left(\lambda_{0}\right) E_{\theta_{1}, \theta_{2}}(z)}{\bar{\lambda}_{0} \theta_{2} \omega_{\lambda_{0}}(z)} .
$$

Now, we write down an important special case of the domination (5.8). Namely, for any $\varphi$ univalent in $\mathbb{D}$ we have

$$
\left\|L_{\varphi, \Lambda, \Theta}\left(\lambda_{0}, \cdot\right)\right\|_{\mathcal{B}_{\Lambda, \Theta}}^{2} \leq k_{\Lambda, \Theta}^{+}\left(\lambda_{0}, \lambda_{0}\right) .
$$

Here, explicit formulas for the norm $\|\cdot\|_{\mathcal{B}_{\Lambda, \Theta}}$ are given by (6.6) and (6.7), and the kernel $L_{\varphi, \Lambda, \Theta}\left(\lambda_{0}, u\right)$ looks like this:

$$
\begin{aligned}
L_{\varphi, \Lambda, \Theta} & \left(\lambda_{0}, u\right) \\
= & \left(\frac{u}{\varphi(u)-\varphi(0)} \frac{\varphi\left(\lambda_{0}\right)-\varphi(0)}{\lambda_{0}}\right)^{\theta_{1}}\left(\frac{u-\lambda_{0}}{\varphi(u)-\varphi\left(\lambda_{0}\right)} \varphi^{\prime}\left(\lambda_{0}\right) \frac{1-\left|\lambda_{0}\right|^{2}}{1-\bar{\lambda}_{0} u}\right)^{\theta_{2}} \frac{\varphi^{\prime}(u)}{\varphi\left(\lambda_{0}\right)-\varphi(u)} \\
& -\frac{1}{\lambda_{0}-u}+\frac{\theta_{2} \bar{\lambda}_{0}}{1-\bar{\lambda}_{0} u} k_{\Lambda, \Theta}^{+}\left(\lambda_{0}, \lambda_{0}\right) .
\end{aligned}
$$

The constant $k_{\Lambda, \Theta}^{+}\left(\lambda_{0}, \lambda_{0}\right)$ admits a representation easier than that given by the limit as $z \rightarrow \lambda_{0}$ in (6.21). Namely, any Möbius shift of variables shows that for any $\lambda_{1}, \lambda_{2} \in \mathbb{D}$ and a Möbius transformation $\omega$ of $\mathbb{D}$ we have

$$
k_{\left\{\omega\left(\lambda_{1}\right), \omega\left(\lambda_{2}\right)\right\},\left\{\theta_{1}, \theta_{2}\right\}}^{+}(z, u)=k_{\left\{\lambda_{1}, \lambda_{2}\right\},\left\{\theta_{1}, \theta_{2}\right\}}^{+}(\omega(z), \omega(u)) .
$$


In particular, applying this formula with

$$
\omega(z)=\frac{\lambda_{0}-z}{1-\bar{\lambda}_{0} z}
$$

we obtain

$$
k_{\left\{0, \lambda_{0}\right\},\left\{\theta_{1}, \theta_{2}\right\}}^{+}\left(\lambda_{0}, \lambda_{0}\right)=k_{\left\{\lambda_{0}, 0\right\},\left\{\theta_{1}, \theta_{2}\right\}}^{+}(0,0)=k_{\left\{0, \lambda_{0}\right\},\left\{\theta_{2}, \theta_{1}\right\}}^{+}(0,0) .
$$

From (6.20) it follows that

$$
\begin{aligned}
k_{\left\{0, \lambda_{0}\right\},\left\{\theta_{2}, \theta_{1}\right\}}^{+}(0,0) & \\
=\frac{1}{\theta_{2}\left|\lambda_{0}\right|^{2}}\{1-( & \left.\int_{0}^{1}\left(1-\left|\lambda_{0}\right|^{2} t\right)^{-\theta_{1}} t^{\theta_{2}-1}(1-t)^{\theta_{1}-1} d t\right)^{-1} \\
& \left.\times \int_{0}^{1}\left(1-\left|\lambda_{0}\right|^{2} t\right)^{1-\theta_{1}} t^{\theta_{2}-1}(1-t)^{\theta_{1}-1} d t\right\},
\end{aligned}
$$

which gives

$$
\begin{aligned}
k_{\Lambda, \Theta}^{+}\left(\lambda_{0}, \lambda_{0}\right)= & \frac{1}{\theta}\left(\int_{0}^{1}\left(1-\left|\lambda_{0}\right|^{2} t\right)^{-\theta_{1}} t^{\theta_{2}-1}(1-t)^{\theta_{1}-1} d t\right)^{-1} \\
& \times \int_{0}^{1}\left(1-\left|\lambda_{0}\right|^{2} t\right)^{-\theta_{1}} t^{\theta_{2}}(1-t)^{\theta_{1}-1} d t
\end{aligned}
$$

$\left(\right.$ for $\left.\Lambda=\left\{0, \lambda_{0}\right\}, \Theta=\left\{\theta_{1}, \theta_{2}\right\}\right)$.

Derivation of Goluzin's inequality. In this subsection, we derive the pointwise estimate (1.8) from a special case of the integral inequality (6.22).

For $\Lambda=\left\{0, \lambda_{0}\right\}$ and $\Theta=\left\{\frac{1}{2}, \frac{1}{2}\right\}$, estimate (6.22) takes the form

$$
\int_{\mathbb{D}}\left|L_{\varphi, \Lambda, \Theta}\left(\lambda_{0}, u\right)\right|^{2} \frac{d A(u)}{|u|\left|\omega_{\lambda_{0}}(u)\right|} \leq k_{\Lambda, \Theta}^{+}\left(\lambda_{0}, \lambda_{0}\right),
$$

where

$$
\begin{aligned}
L_{\varphi, \Lambda, \Theta}\left(\lambda_{0}, u\right)= & \left(\frac{u}{\varphi(u)} \frac{\varphi\left(\lambda_{0}\right)}{\lambda_{0}}\right)^{1 / 2}\left(\frac{u-\lambda_{0}}{\varphi(u)-\varphi\left(\lambda_{0}\right)} \varphi^{\prime}\left(\lambda_{0}\right) \frac{1-\left|\lambda_{0}\right|^{2}}{1-\bar{\lambda}_{0} u}\right)^{1 / 2} \frac{\varphi^{\prime}(u)}{\varphi\left(\lambda_{0}\right)-\varphi(u)} \\
& -\frac{1}{\lambda_{0}-u}+\frac{\theta_{2} \bar{\lambda}_{0}}{1-\bar{\lambda}_{0} u} k_{\Lambda, \Theta}^{+}\left(\lambda_{0}, \lambda_{0}\right),
\end{aligned}
$$

provided $\varphi(0)=0$. Since the left-hand side of (6.24) is the norm of $L_{\varphi, \Lambda, \Theta}\left(\lambda_{0}, \cdot\right)$ in $\mathcal{B}_{\Lambda, \Theta}$, we see that

$$
\left|L_{\varphi, \Lambda, \Theta}\left(\lambda_{0}, \lambda_{0}\right)\right| \leq\left[k_{\Lambda, \Theta}^{-}\left(\lambda_{0}, \lambda_{0}\right) k_{\Lambda, \Theta}^{+}\left(\lambda_{0}, \lambda_{0}\right)\right]^{1 / 2} .
$$

An explicit calculation shows that

$$
L_{\varphi, \Lambda, \Theta}\left(\lambda_{0}, \lambda_{0}\right)=-\frac{1}{4}\left(\frac{\varphi^{\prime \prime}\left(\lambda_{0}\right)}{\varphi^{\prime}\left(\lambda_{0}\right)}-2 \frac{\varphi^{\prime}\left(\lambda_{0}\right)}{\varphi\left(\lambda_{0}\right)}+\frac{2}{\lambda_{0}}+\frac{2 \bar{\lambda}_{0}}{1-\left|\lambda_{0}\right|^{2}}-\frac{2 \bar{\lambda}_{0}}{1-\left|\lambda_{0}\right|^{2}} k_{\Lambda, \Theta}^{+}\left(\lambda_{0}, \lambda_{0}\right)\right) .
$$

By Proposition 3.3, we have

$$
k_{\Lambda, \Theta}^{-}\left(\lambda_{0}, \lambda_{0}\right)=\frac{1}{4}\left|\lambda_{0}\right|^{2} \frac{k_{\Lambda, \Theta}^{+}\left(\lambda_{0}, \lambda_{0}\right)}{\left(1-\left|\lambda_{0}\right|^{2}\right)^{2}} .
$$

Finally, formula (6.23) yields

$$
k_{\Lambda, \Theta}^{+}\left(\lambda_{0}, \lambda_{0}\right)=2\left|\lambda_{0}\right|^{-2}\left(1-\left(\int_{0}^{1} \frac{d t}{\sqrt{t(1-t)\left(1-\left|\lambda_{0}\right|^{2} t\right)}}\right)^{-1} \cdot \int_{0}^{1} \sqrt{\frac{1-\left|\lambda_{0}\right|^{2} t}{t(1-t)}} d t\right) .
$$


If $E(z)$ and $K(z)$ denote the standard complete elliptic integrals

$$
E(z)=\int_{0}^{1} \sqrt{\frac{1-z t^{2}}{1-t^{2}}} d t \text { and } K(z)=\int_{0}^{1} \frac{d t}{\sqrt{\left(1-t^{2}\right)\left(1-z t^{2}\right)}}
$$

we obtain

$$
k_{\Lambda, \Theta}^{+}\left(\lambda_{0}, \lambda_{0}\right)=\frac{2}{\left|\lambda_{0}\right|^{2}}\left(1-\frac{E\left(\left|\lambda_{0}\right|^{2}\right)}{K\left(\left|\lambda_{0}\right|^{2}\right)}\right) .
$$

Substituting this in the preceding formulas, we arrive at the following pointwise estimate for $\varphi \in S$ :

$$
\begin{aligned}
& \left|\lambda_{0} \frac{\varphi^{\prime \prime}\left(\lambda_{0}\right)}{\varphi^{\prime}\left(\lambda_{0}\right)}-2 \frac{\lambda_{0} \varphi^{\prime}\left(\lambda_{0}\right)}{\varphi\left(\lambda_{0}\right)}+2+\frac{2\left|\lambda_{0}\right|^{2}-4}{1-\left|\lambda_{0}\right|^{2}}+4 \frac{E\left(\left|\lambda_{0}\right|^{2}\right)}{K\left(\left|\lambda_{0}\right|^{2}\right)} \frac{1}{1-\left|\lambda_{0}\right|^{2}}\right| \\
& \quad \leq 4\left(1-\frac{E\left(\left|\lambda_{0}\right|^{2}\right)}{K\left(\left|\lambda_{0}\right|^{2}\right)}\right) .
\end{aligned}
$$

Originally, it appeared in [6, Chapter IV, $\S 3$, formula (30)], and it is equivalent to the pointwise estimate (1.8) for functions of class $\Sigma$.

\section{REFERENCES}

[1] N. Abuzyarova and H. Hedenmalm, Branch point area methods in conformal mapping, J. Anal. Math. (to appear).

[2] S. Bergman and M. Schiffer, Kernel functions and conformal mapping, Compositio Math. 8 (1951), 205-249. MR0039812 (12:602c)

[3] J. Bognár, Indefinite inner product spaces, Ergeb. Math. Grenzgeb., vol. 78, Springer-Verlag, New York-Heidelberg, 1974. MR0467261 (57:7125)

[4] L. Carleson, A representation formula for the Dirichlet integral, Math. Z. 73 (1960), 190-196. MR0112958 (22:3803)

[5] P. L. Duren, Univalent functions, Grundlehren Math. Wiss., vol. 259, Springer-Verlag, New York, 1983. MR0708494 (85j:30034)

[6] G. M. Goluzin, Geometric theory of functions of a complex variable, "Nauka," Moscow, 1966; English transl., Transl. Math. Monogr., vol. 26, Amer. Math. Soc., Providence, RI, 1969. MR0219714 (36:2793) MR0247039 (40:308)

[7] H. Hedenmalm and S. Shimorin, Weighted Bergman spaces and the integral means spectrum of conformal mappings, Duke Math. J. 127 (2005), 341-393. MR2130416 (2005m:30010)

[8] N. A. Lebedev, The area principle in the theory of univalent functions, "Nauka", Moscow, 1975. (Russian) MR0450540 (56:8834)

[9] Z. Nehari, Inequalities for the coefficients of univalent functions, Arch. Rational Mech. Anal. 34 (1969), 301-330. MR0247061 (40:330)

[10] Ch. Pommerenke, Univalent functions. With a chapter on quadratic differentials by Gerd Jensen, Studia Math./Math. Lehrbücher, Band XXV, Vandenhoeck and Ruprecht, Göttingen, 1975. MR $0507768(58: 22526)$

[11] H. Prawitz, Uber Mittelwerte analytischer Funktionen, Arkiv Mat. Astronomy Fysik 20A (19271928), no. 6, 1-12.

[12] S. Shimorin, A multiplier estimate of the Schwarzian derivative of univalent functions, Int. Math. Res. Not. 2003, no. 30, 1623-1633. MR1979583(2004c:30031)

Department of Mathematics, Royal Institute of Technology, 10044 Stockholm, Sweden E-mail address: shimorin@math.kth.se

Received 10/OCT/2005

Originally published in English 